\title{
pandeo en régimen elastoplástico de paneles metálicos rigidizados longitudinalmente
}

\author{
José Ramón Atienza Reales, \\ Dr. Ingeniero de Caminos*
}

Marcos Jesús Pantaleón Prieto
Dr. Ingeniero de Caminos**

sinopsis

En este artículo exponemos una técnica matricial, de análisis armónico, que permite analizar el pandeo en régimen elastoplástico de paneles rigidizados solicitados por cargas longitudinales.

Recordemos que existen dos métodos básicos para el estudio del pandeo de elementos metálicos comprimidos: el de bifurcación de equilibrio, que permite determinar las cargas CRITICAS de los sistemas PERFECTOS, y el de divergencia de equilibrio, que permite determinar las cargas de AGOTAMIENTO de los sistemas REALES. La diferencia básica entre ambos métodos radica en que el primero analiza el pandeo como un problema de equilibrio indiferente (infinitas soluciones), mientras que el segundo analiza el pandeo como un problema de resistencia (punto más alto del diagrama carga-deformación).

Nosotros analizaremos el pandeo del panel considerándolo como un problema de bifurcación de equilibrio. Por consiguiente, supondremos que las cargas exteriores están perfectamente centradas en el plano medio del panel, y despreciaremos todas sus imperfecciones geométricas.

En primer lugar, vamos a estudiar el pandeo en régimen elástico de estos sistemas estructurales, y a continuación, analizaremos la influencia que tienen las tensiones residuales de soldadura en el valor de la menor carga crítica, en los supuestos de que el pandeo tenga lugar en régimen elástico o en régimen elastoplástico.

* Catedrático de Estructuras y Puentes Metálicos de la E.T.S. de Ingenieros de Caminos, Canales y Puertos de la Universidad de Santander.

* Profesor Ayudante de Estructuras y Puentes Metálicos de la E.T.S. de Ingenieros de Caminos Canales y Puertos de la Universidad de Santander. 


\section{Introducción}

Los paneles metálicos rigidizados longitudinalmente encuentran aplicación dentro del campo de las ingenierías civil, naval y aeronáutica.

A modo de ejemplo, en la figura 1, hemos representado un pilono de un puente colgante formado por dos columnas metálicas, de sección en cajón multicelular, que se encuentran arriostradas entre sí con diagonales en cruz de S. Andrés. Para evitar el pandeo de las paredes de las columnas debemos rigidizarlas, esto se consigue soldando diafragmas transversales y rigidizadores longitudinales que, como se indica en la figura, pueden ser abiertos o cerrados.

Al actuar las cargas que solicitan el sistema estructural, del que el panel es un elemento constituyente, se inducen en éste ciertas deformaciones y esfuerzos cuya magnitud puede evaluarse fácilmente aplicando las técnicas de análisis estructural de todos conocidas.

Para estudiar el pandeo del panel (bifurcación de equilibrio) debemos idealizar el problema suponiendo yue, durante la actuación de las cargas exteriores, cada una de las chapas que componen el panel permanece plana, y que, además, los únicos esfuerzos inducidos por la acción exterior son esfuerzos $\mathrm{N}_{x}$ que se mantienen constantes dentro de cada chapa (figura 2). No es necesario que todas las chapas tengan la misma solicitación, basta con que el sistema de cargas quede definido en función de un parámetro $\lambda$ que, al crecer, aumenta la solicitación de todas ellas.

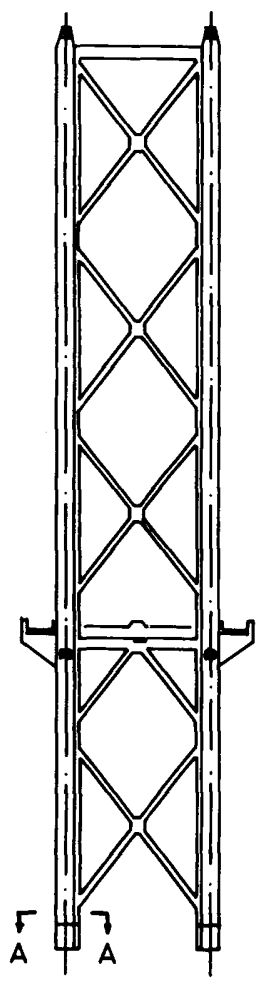

Pilono de puente colgante

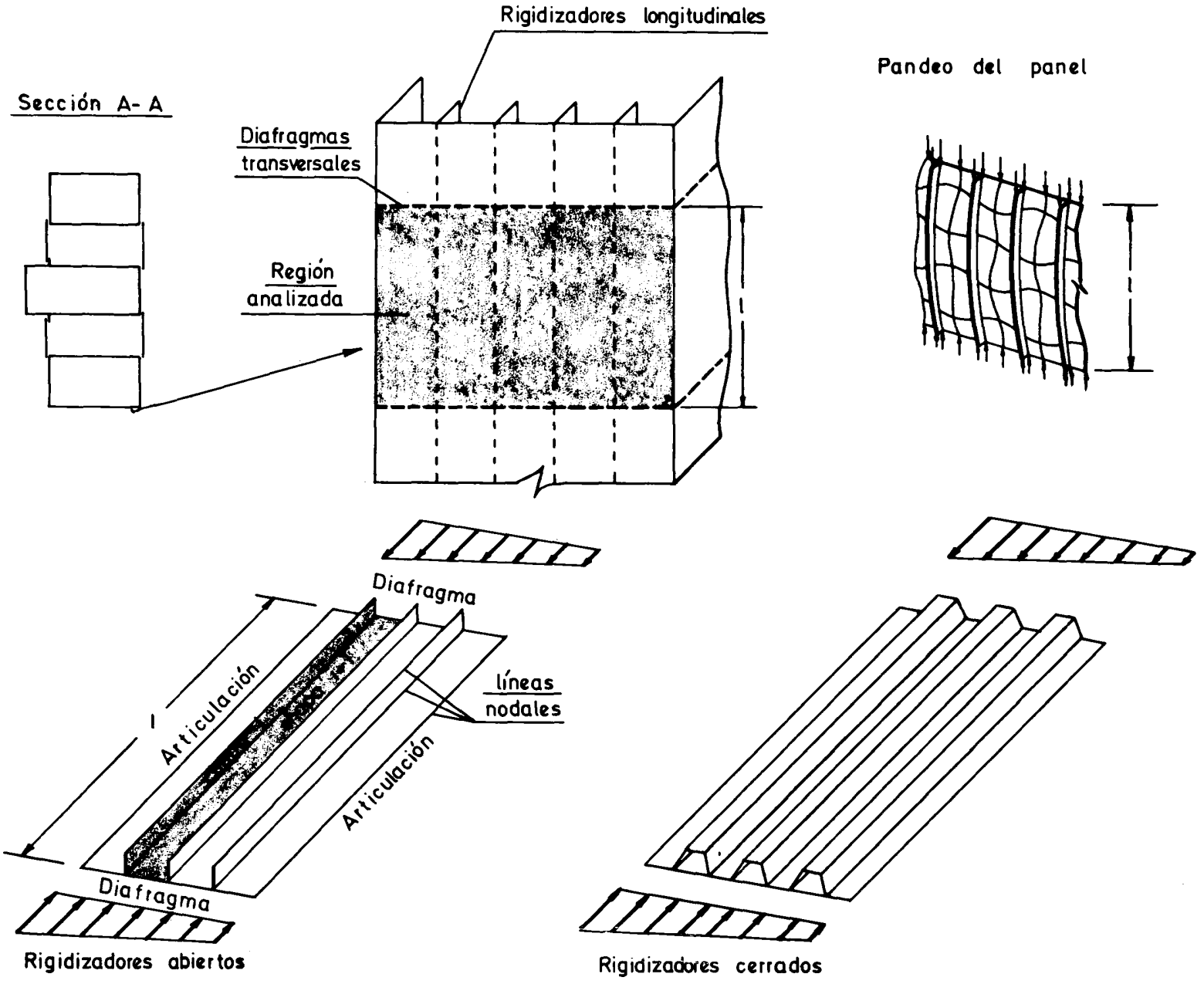

Figura 1 


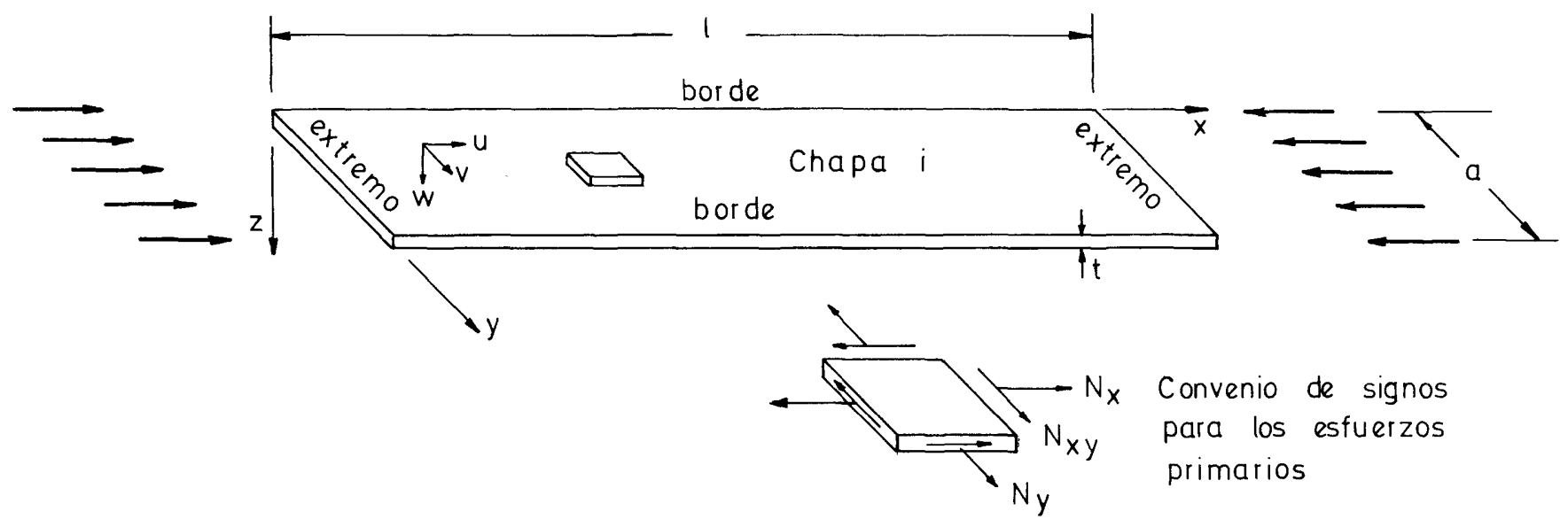

Figura 2

Con el fin de simplificar la nomenclatura, llamaremos GEOMETRIA INICIAL a la configuración de equilibrio deformada debida a la actuación de las cargas exteriores, así mismo, denominaremos ESFUERZOS PRIMARIOS a los esfuerzos inducidos por la actuación de dichas cargas.

La pregunta que nos hacemos cuando estudiamos el pandeo del panel, aplicando un método de bifurcación de equilibrio, es la siguiente:

¿Existe la posibilidad de que el panel rigidizado esté, también, en equilibrio, en infinitas soluciones deformadas muy próximas a la llamada geometría inicial?

Para contestar esta pregunta debemos integrar las ecuaciones diferenciales que, para cada una de sus chapas, establecen el equilibrio en una posición ligeramente deformada.

Para facilitar la integración de estas ecuaciones diferenciales, nos limitaremos a analizar el problema. en el supuesto de que en los dos extremos del panel rigidizado existan diafragmas, ya que, de este modo, se pueden satisfacer las condiciones de contorno en los extremos de las chapas con soluciones en serie de Levi.

Al imponer las condiciones de contorno en los bordes longitudinales, en los que las distintas chapas se ensamblan entre sí (condiciones de equilibrio y compatibilidad de movimientos), obtenemos un sistema de ecuaciones homogéneo, del que algunos de sus coeficientes son funciones trascendentes del parámetro $\lambda, y$ otros, son constantes. Este sistema de ecuaciones admite infinitas soluciones para aquellos valores de $\lambda$ que anulan el determinante de sus coeficientes. Nuestro objetivo es, precisamente, evaluar los valores de $\lambda=\lambda_{c}$ (valores críticos) para los que el sistema estructural admite infinitas soluciones al problema del equilibrio.

\section{I.1. Sistema de ecuaciones diferenciales que establecen el equilibrio de la chapa en una posición ligeramente deformada}

En el momento del pandeo, las chapas que componen el panel rigidizado, inicialmente planas, sufren ligeros movimientos que inducen unos esfuerzos secundarios.

Cuando la chapa experimenta corrimientos transversales a su plano medio $w^{*}(x, y)$ los esfuerzos secundarios inducidos son de flexión: 


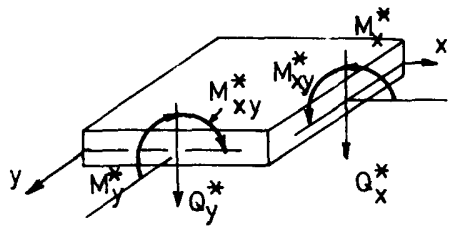

Figura 3

$$
\begin{aligned}
& M^{*}=-D\left(\frac{\partial^{2} w^{*}(x, y)}{\partial x^{2}}+v \frac{\partial^{2} w^{*}(x, y)}{\partial y^{2}}\right) \\
& M^{*} y=-D\left(\frac{\partial^{2} w^{*}(x, y)}{\partial y^{2}}+v \frac{\partial^{2} w^{*}(x, y)}{\partial x^{2}}\right) \\
& M_{x}^{*} y=D(1-v) \frac{\partial^{2} w^{*}(x, y)}{\partial x} \partial y \\
& Q^{*}=-D\left(\frac{\partial^{3} w^{*}(x, y)}{\partial x^{3}}+\frac{\partial^{3} w^{*}(x, y)}{\partial x y^{2}}\right) \\
& Q^{*} y=-D\left(\frac{\partial^{3} w^{*}(x, y)}{\partial x^{2} \partial y}+\frac{\partial^{3} w^{*}(x, y)}{\partial y^{3}}\right)
\end{aligned}
$$

mientras que si los corrimientos están contenidos en el plano medio de la chapa $u^{*}(x, y), v^{*}(x, y)$, los esfuerzos secundarios inducidos son de membrana:

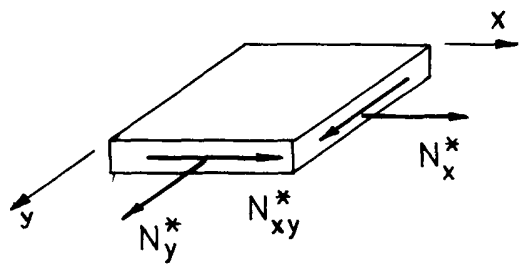

Figura 4

$$
\begin{aligned}
& N^{*}=\frac{E t}{1-v^{2}}\left(\frac{\partial u^{*}(x, y)}{\partial x}+v \frac{\partial v^{*}(x, y)}{\partial y}\right) \\
& N^{*} y=\frac{E t}{1-v^{2}}\left(\frac{\partial v^{*}(x, y)}{\partial y}+v \frac{\partial u^{*}(x, y)}{\partial x}\right) \\
& N^{*} y=\frac{E t}{2(1+v)}\left(\frac{\partial u^{*}(x, y)}{\partial y}+\frac{\partial v^{*}(x, y)}{\partial x}\right)
\end{aligned}
$$

Las ecuaciones diferenciales que establecen el equilibrio en una posición ligeramente deformada, en la que existen ambos tipos de esfuerzos (esfuerzos primarios y secundarios), son:

a) Comportamiento placa (10)

$$
D\left(\frac{\partial^{4} w^{*}}{\partial x^{4}}+2 \frac{\partial^{4} w^{*}}{\partial x^{2} \partial y^{2}}+\frac{\partial^{4} w^{*}}{\partial y^{4}}\right)-N x \frac{\partial^{2} w^{*}}{\partial x^{2}}=0
$$

b) Comportamiento laja (11)

$$
\begin{aligned}
& 2\left[1-\left(1-v^{2}\right) \frac{N x}{E t}\right] \frac{\partial^{2} u^{*}}{\partial x^{2}}+(1-v) \frac{\partial^{2} u^{*}}{\partial y^{2}}+(1+v) \frac{\partial^{2} v^{*}}{\partial x \partial y}=0 \\
& 2 \frac{\partial^{2} v^{*}}{\partial y^{2}}+(1-v)\left[1-2(1+v) \frac{N x}{E t}\right] \frac{\partial^{2} v^{*}}{\partial x^{2}}+(1+v) \frac{\partial^{2} u^{*}}{\partial x \partial y}=0
\end{aligned}
$$

donde:

$D=$ rigidez a flexión de la chapa $=\frac{\mathrm{Et}^{3}}{12\left(1-v^{2}\right)}$

$\mathrm{E}=$ módulo de Young

$v=$ coeficiente de Poisson

$N_{x}=$ esfuerzo primario que solicita a la chapa

$w^{*}, u^{*}, v^{*}=$ corrimientos, en el momento del pandeo, en la dirección de los ejes cartesianos $x, y, z$. 
Las expresiones anteriores se han deducido suponiendo que, en el momento del pandeo, los movimientos transversales al plano medio de la chapa son reducidos. Esto nos ha permitido aplicar la teoría lineal de la flexión de placas delgadas y desacoplar el comportamiento de la chapa como placa del comportamiento como laja.

\section{I.2. Integración del sistema de ecuaciones}

Una solución en serie de Levi de la forma:

$$
\begin{aligned}
& w^{*}(x, y)=\sum_{n=1}^{\infty} w_{n}(y) \operatorname{sen} \frac{n \pi x}{1} \\
& u^{*}(x, y)=\sum_{n=1}^{\infty} u_{n}(y) \cos \frac{n \pi x}{1} \\
& v^{*}(x, y)=\sum_{n=1}^{\infty} v_{n}(y) \operatorname{sen} \frac{n \pi x}{1}
\end{aligned}
$$

satisface automáticamente las condiciones de contorno en los extremos de la chapa, en los que existen diafragmas. Estas condiciones de contorno son:

para $x=0, y, x=1$

$$
\begin{aligned}
& \left\{\begin{array}{l}
w^{*}(x, y)=0 \\
\frac{\partial^{2} w^{*}(x, y)}{\partial x^{2}}=0
\end{array} \quad \text { dado que } M_{x}^{*}=0\right. \\
& \left\{\begin{array}{l}
v^{*}(x, y)=0 \\
\frac{\partial u^{*}(x, y)}{\partial x}=0
\end{array} \text { dado que } N_{x}^{*}=0\right.
\end{aligned}
$$

para que $w^{*}(x, y)$ verifique la ecuación $1-1$, la función $W_{n}(y)$ debe ser:

para $N x>0 ; W_{n}(y)=A_{n} \operatorname{ch} \beta_{n} y \cos \rho_{n} y+B_{n} \operatorname{ch} \beta_{n} y \operatorname{sen} \rho_{n} y+C_{n} \operatorname{sh} \beta_{n} y \cos \rho_{n} y+D_{n} \operatorname{sh} \beta_{n} y \operatorname{sen} \rho_{n} y$

$$
\begin{array}{ll}
\text { para } N x=0 ; W_{n}(y)=A_{n} \operatorname{sh} \beta_{n} y & +C_{n} \beta_{n} y \operatorname{sh} \beta_{n} y+D_{n} \beta_{n} y \operatorname{ch} \beta_{n} y \\
\text { para } N x<\left(\frac{n \pi}{1}\right)^{2} D ; W_{n}(y)=A_{n} \operatorname{sh} \beta_{n} y & +B_{n} \operatorname{ch} \beta_{n} y+C_{n} \operatorname{sh} \rho_{n} y+D_{n} \operatorname{ch} \rho_{n} y \\
\text { para } N x=\left(\frac{n \pi}{1}\right)^{2} D ; W_{n}(y)=A_{n} \operatorname{sh} \beta_{n} y+B_{n} \operatorname{ch} \beta_{n} y+ & +C_{n} \beta_{n} y+D_{n} \\
\text { para } N x>\left(\frac{n \pi}{1}\right)^{2} D ; W_{n}(y)=A_{n} \operatorname{sh} \beta_{n} y+B_{n} \operatorname{ch} \beta_{n} y+ & +C_{n} \operatorname{sen} \rho_{n} y+D_{n} \cos \rho_{n} y
\end{array}
$$


análogamente, para que $u^{*}(x, y)$ y $v^{*}(x, y)$ verifiquen las ecuaciones $I-2,3$, las funciones $U_{n}(y)$ y $V_{n}(y)$ deben ser:

para $N x \neq 0\left[\begin{array}{l}U_{n}(y)=F_{n} \operatorname{sh} \delta_{n} y+G_{n} \operatorname{ch} \delta_{n} y+H_{n} \operatorname{sh} \gamma_{n} y+M_{n} \operatorname{ch} \gamma_{n} y \\ \left.V_{n}(y)=\frac{1}{n \pi}\left[\delta_{n}\left(F_{n} \operatorname{ch} \delta_{n} y+G_{n} \operatorname{sh} \delta_{n} y\right)+\gamma_{n} H_{n} \operatorname{ch} \gamma_{n} y+M_{n} \operatorname{sh} \gamma_{n} y\right)\right]\end{array}\right.$
para $N x=0\left[\begin{array}{l}U_{n}(y)=F_{n} \operatorname{sh} \delta_{n} y+G_{n} \operatorname{ch} \delta_{n} y+\delta_{n}\left(H_{n} y \operatorname{sh} \delta_{n} y+M_{n} y \operatorname{ch} \delta_{n} y\right) \\ V_{n}(y)=\left(G_{n}-H_{n}\left(\frac{3-V}{1+V}\right)\right) \operatorname{sh} \delta_{n} y+\left(F_{n}-M_{n}\left(\frac{3-V}{1+V}\right)\right) \operatorname{ch} \delta_{n} y+M_{n} \delta_{n} y \operatorname{sh} \delta_{n} y+H_{n} \delta_{n} y \operatorname{ch} \delta_{n} y\end{array}\right.$

los coeficientes, $\beta_{n}, Q_{n}, \delta_{n}$ y $\gamma_{n}$ son función de la solicitación $\mathrm{N}_{x}$, de la geometría de la chapa y del número entero $n(n=1,2,3, \ldots)$. Los valores de estos coeficientes se han tabulado en el apéndice 1 .

Las expresiones 1-4, 5 y 6 definen la deformada de cada una de las chapas en forma de una serie infinita, función de un conjunto de constantes $\mathrm{A}_{n}, \mathrm{~B}_{n}, \mathrm{C}_{n}, \mathrm{D}_{n}, \mathrm{~F}_{n}, \mathrm{G}_{n}, \mathrm{H}_{n}, \mathrm{y} \mathrm{M}_{n}$, que deben determinarse imponiendo las condiciones de contorno en los bordes longitudinales $(y=0, y=a)$ en los que las distintas chapas se ensamblan entre sí.

Estas condiciones de contorno son: las ecuaciones de equilibrio y compatibilidad de movimiento en las líneas nodales. Al no haber cargas aplicadas en las líneas nodales, las condiciones de contorno conducen a un sistema homogéneo, con ocho ecuaciones por cada una de las chapas que componen el panel rigidizado. Este sistema tiene la forma general:

$$
\begin{aligned}
& \sum_{n=1}^{\infty}\left(\alpha_{n}^{8 i-7,8 j-7} A_{n, j}+\alpha_{n}^{8 i-7,8 j-6} B_{n, j}+\cdots \cdots \cdots \cdots \cdots+\alpha_{n}^{8 i-7,8 j} M_{n, j}\right) \operatorname{sen} \frac{n \pi x}{l}=0 \\
& \sum_{n=1}^{\infty}\left(\alpha_{n}^{8 i-6,8 j-7} A_{n, j}+a_{n}^{8 i-6,8 j-6} B_{n, j}+\cdots \ldots \ldots \ldots \ldots \ldots+\alpha_{n}^{8 i-6,8 j} M_{n, j}\right) \operatorname{sen} \frac{n \pi x}{1}=0 \\
& \sum_{n=1}^{\infty}\left(a_{n}^{8 i-5,8 j-7} A_{n, j}+a_{n}^{8 i-5,8 j-6} B_{n, j}+\cdots \cdots \cdots \cdots \cdots \cdots+a_{n}^{8 i-5,8 j} M_{n, j}\right) \operatorname{sen} \frac{n \pi x}{1}=0 \\
& \sum_{n=1}^{\infty}\left(a_{n}^{8 i-4,8 j-7} A_{n, j}+a^{8 i-4,8 j-6} B_{n, j}+\cdots \cdots \cdots \cdots \cdots \cdots+\alpha_{n}^{8 i-4,8 j} M_{n, j}\right) \cos \frac{n \pi x}{1}=0 \\
& \sum_{n=1}^{\infty}\left(a_{n}^{8 i-3,8 j-7} A_{n, j}+a_{n}^{8 i-3,8 j-6} B_{n, j}+\cdots \cdots \cdots \cdots \cdots \cdots \cdots+a_{n}^{8 i-3,8 j} M_{n, j}\right) \operatorname{sen} \frac{n \pi x}{l}=0 \\
& \sum_{n=1}^{\infty}\left(\alpha_{n}^{8 i-2,8 j-7} A_{n, j}+a_{n}^{8 i-2,8 j-6} B_{n, j}+\cdots \cdots \cdots \ldots \ldots \ldots+\alpha_{n}^{8 i-2,8 j} M_{n, j}\right) \operatorname{sen} \frac{n \pi x}{1}=0 \\
& \sum_{n=1}^{\infty}\left(a_{n}^{8 i-1,8 j-7} A_{n, j}+a_{n}^{8 i-1,8 j-6} B_{n, j}+\cdots \cdots \cdots \cdots \cdots \cdots+\alpha_{n}^{8 i-1,8 j} M_{n, j}\right) \operatorname{sen} \frac{n \pi x}{l}=0 \\
& \sum_{n=1}^{\infty}\left(\alpha_{n}^{8 i, 8 j-7} A_{n, j}+\alpha_{n}^{8 i, 8 j-6} B_{n, j}+\cdots \cdots \cdots \ldots \ldots \ldots+\alpha_{n}^{8 i, 8 j} M_{n, j}\right) \cos \frac{n \pi x}{1}=0
\end{aligned}
$$

donde:

$i, j=1,2, \ldots, r$

$r=$ número de chapas que componen el panel. 
Los términos $\alpha_{n}$, son, en general, funciones trascendentes del parámetro $\lambda$ y del número entero $\mathrm{n}$. Para valores arbitrarios de $\mathrm{n}$ y $\lambda$ el sistema de ecuaciones se satisface únicamente cuando son nulos los coeficientes $\mathrm{A}_{n, j}, \mathrm{~B}_{n, j}, \mathrm{C}_{n, j}, \mathrm{D}_{n, j}, \mathrm{~F}_{n, j}, \mathrm{G}_{n, j}, \mathrm{H}_{n, j}$ y $\mathrm{M}_{n, j}$ (para $\forall_{n,}$ y $\mathrm{j}=1,2, \ldots, \mathrm{r}$ ), es decir, el panel rigidizado está en equilibrio, solamente, si conserva su geơmetría inicial. No obstante, es fácil demostrar que el sistema homogéneo admite infinitas soluciones deformadas, para aquellos valores de $n$ y $\lambda$ que anulan el determinante:

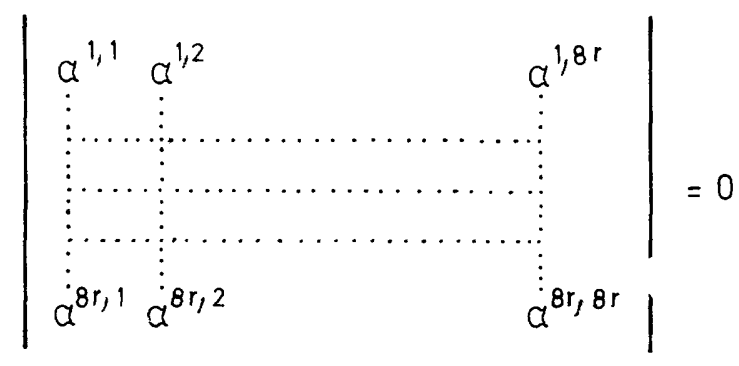

En realidad, para cada número entero $\mathrm{n}$ existen infinitos valores de $\lambda=\lambda_{c}$ que anulan dicho determinante. A nosotros sólo nos interesa determinar el menor de todos los valores de $\lambda=\lambda_{c 1}$ para el que existen infinitas soluciones al problema del equilibrio, ya que se puede demostrar que, para $\lambda<\lambda_{c 1}$, la llamada geometría inicial es una configuración de equilibrio estable, mientras que, para $\lambda>\lambda_{c 1}$, la geometría inicial es una configuración de equilibrio inestable.

La geometría de equilibrio deformada en el momento del pandeo (modo de pandeo), viene definida longitudinalmente por $\mathrm{n} \mathrm{y}$, transversalmente, por los coeficientes $\mathrm{A}_{n j}, \mathrm{~B}_{n j}, \ldots y \mathrm{M}_{n j}$.

El número $n$ (fórmulas $1-4,5$ y 6 ) define el número de semiondas senoidales en que queda dividida la longitud del panel en el momento del pandeo.

Los coeficientes $A_{n, j}, B_{n j,}, \ldots$ y $\mathrm{M}_{n, j}$, se pueden evaluar sustituyendo $\lambda=\lambda_{c}$ en el sistema 1-9. Como se trata de un sistema homogéneo y su determinante es nulo, se pueden despejar todos los coeficientes en función de uno de ellos, que queda como constante arbitraria, pudiendo tomar cualquier valor. Se trata, pues, de una geometría de equilibrio deformada, definida en su forma, pero no en magnitud (infinitas soluciones).

\section{Método armónico en rigidez para el estudio del pandeo en régimen elástico de paneles rigidizados}

En el apartado anterior hemos puesto de manifiesto que los paneles rigidizados, solicitados por cargas longitudinales contenidas en su plano medio, pandean sinusoidalmente, de forma que la longitud total del panel queda dividida en un número entero de semiondas. En consecuencia, en el momento del pandeo, la deformada de cada una de las chapas que componen el panel, así como los esfuerzos de interacción entre las chapas, varían, también, de forma sinusoidal.

Esta circunstancia es la que nos va a permitir aplicar un método armónico en rigidez para el estudio del pandeo de los paneles rigidizados.

La técnica que se aplica consiste en analizar, en primer lugar, cada una de las chapas que componen el sistema estructural, buscando la relación que existe entre un sistema de cargas sinusoidales aplicadas en sus dos bordes y los movimientos sinusoidales que dichas cargas provocan. 
Una vez que se ha deducido la relación anterior (matriz de rigidez de la chapa aislada), procedemos a ensamblar las chapas entre sí, en las líneas nodales, estableciendo las ecuaciones de compatibilidad y equilibrio.

De esta forma, conseguimos relacionar mediante un sistema de ecuaciones, los movimientos sinusoidales de las líneas nodales con las cargas exteriores que actúan en dichas líneas nodales. Pero, dado que no hay cargas exteriores aplicadas en las líneas nodales, el sistema de ecuaciones que obtenemos es un sistema homogéneo. Sus soluciones no triviales son las que definen las cargas críticas y sus modos de pandeo asociados.

\section{II.1. Matriz de rigidez de la chapa referida a sus ejes locales}

Analizaremos el problema, en primer lugar, en el supuesto de que las cargas aplicadas en los bordes longitudinales movilizan únicamente esfuerzos de flexión. A continuación, lo analizaremos en el supuesto de que movilizan únicamente esfuerzos de membrana.

a) Comportamiento placa.

Con referencia a la figura 5 podemos afirmar:

$$
\begin{aligned}
& m_{1}=\bar{m}_{1} \operatorname{sen} \frac{n \pi x}{l}=-(M y)_{y}=0 \\
& f_{1}=\bar{f}_{1} \operatorname{sen} \frac{n \pi x}{l}=-(R y)_{y}=0 \\
& m_{2}=\bar{m}_{2} \operatorname{sen} \frac{n \pi x}{l}=(M y)_{y}=a \\
& f_{2}=\bar{f}_{2} \operatorname{sen} \frac{n \pi x}{l}=(R y)_{y=a}
\end{aligned}
$$

$$
\text { donde } \begin{aligned}
: M y & =-D\left(\frac{\partial^{2} w^{*}(x, y)}{\partial y^{2}}+v \frac{\partial^{2} w^{*}(x, y)}{\partial x^{2}}\right) \\
R y & =Q y-\frac{\partial M_{y x}}{\partial x}=-D\left[\frac{\partial^{3} w^{*}(x, y)}{\partial y^{3}}+(2-V) \frac{\partial^{3} w^{*}(x, y)}{\partial x^{2} \partial y}\right]
\end{aligned}
$$

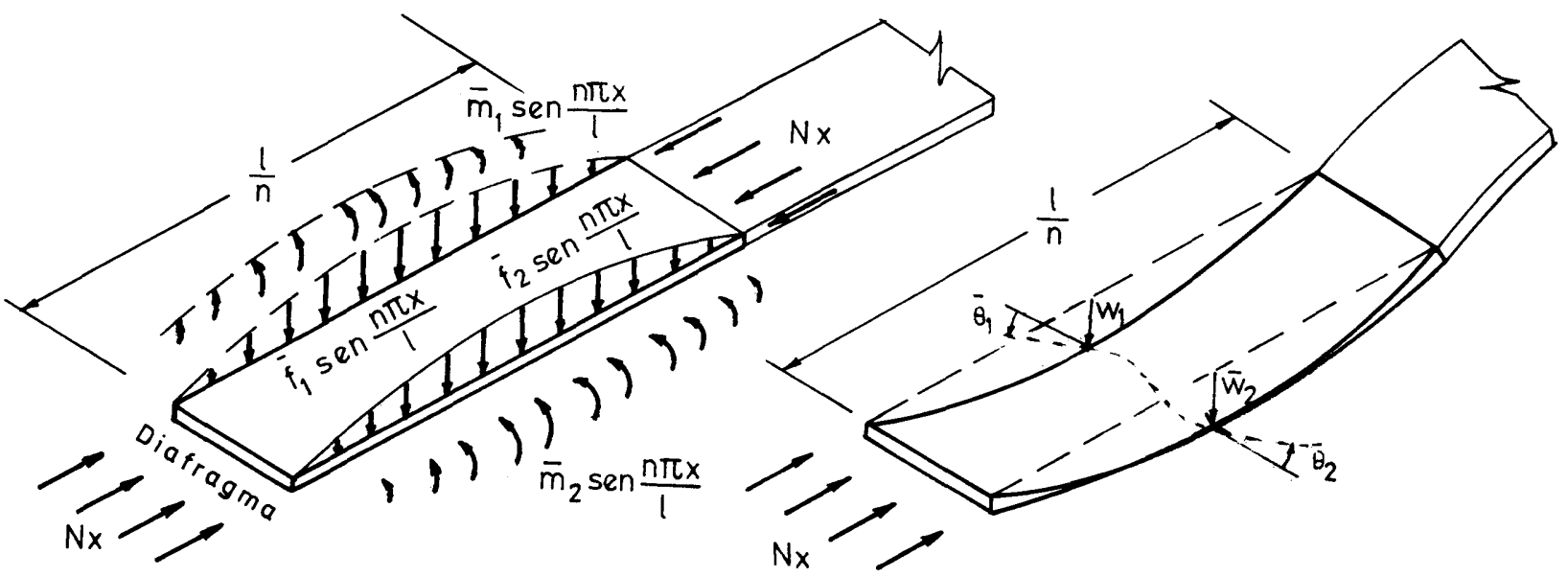

Figura 5 
La deformada transversal de la chapa $\mathrm{w}^{*}(\mathrm{x}, \mathrm{y})$ viene definida por la expresión $\mathrm{I}-4$, y los coeficientes indeterminados $\mathrm{A}_{n}, \mathrm{~B}_{n}, \mathrm{C}_{n}, \mathrm{y} \mathrm{D}_{n}$, de dicha expresión, se pueden evaluar imponiendo las condiciones de contorno en los dos bordes longitudinales:

$$
\begin{aligned}
& \text { para } y=0\left\{\begin{array}{l}
w^{*}(x, y)=\bar{w}_{1} \operatorname{sen} \frac{n \pi x}{l} \\
\theta^{*}(x, y)=\frac{\partial w^{*}(x, y)}{\partial y}=-\bar{\theta}_{1} \operatorname{sen} \frac{n \pi x}{l}
\end{array}\right. \\
& \text { para } y=a\left\{\begin{array}{l}
w^{*}(x, y)=\bar{w}_{2} \operatorname{sen} \frac{n \pi x}{l} \\
\theta^{*}(x, y)=\frac{\partial w^{*}(x, y)}{\partial y}=-\bar{\theta}_{2} \operatorname{sen} \frac{n \pi x}{l}
\end{array}\right.
\end{aligned}
$$

Ordenando matricialmente las expresiones II-1, podemos escribir:

$$
\left[\begin{array}{l}
\bar{m}_{1} \\
\bar{f}_{1} \\
\bar{m}_{2} \\
\bar{f}_{2}
\end{array}\right]=\left[\begin{array}{llll}
p_{1,1} & p_{1,2} & p_{1,3} & p_{1,4} \\
p_{2,1} & p_{2,2} & p_{2,3} & p_{2,4} \\
p_{3,1} & p_{3,2} & p_{3,3} & p_{3,4} \\
p_{4,1} & p_{4,2} & p_{4,3} & p_{4,4}
\end{array}\right]\left[\begin{array}{c}
\bar{\theta}_{1} \\
\bar{w}_{1} \\
\bar{\theta}_{2} \\
\bar{w}_{2}
\end{array}\right]
$$

cuyos coeficientes $P_{i j}(i, j=1,2,3$ y 4 ) hemos tabulado en el apéndice 3 . Estos coeficientes son funciones trascendentes de la solicitación, de la geometría de la chapa y del número entero $n$ ( $n=1,2,3, \ldots$ ).

b) Comportamiento laja.

Con referencia a la figura 6 podemos afirmar:

$$
\begin{aligned}
& n_{1}=\bar{n}_{1} \operatorname{sen} \frac{n \pi x}{1}=-(N y)_{y}=0 \\
& r_{1}=\bar{r}_{1} \cos \frac{n \pi x}{1}=-(N x y)_{y=0} \\
& n_{2}=\bar{n}_{2} \operatorname{sen} \frac{n \pi x}{1}=(N y)_{y}=a \\
& r_{2}=\bar{i}_{2} \cos \frac{n \pi x}{1}=(N x y)_{y=a}
\end{aligned}
$$

donde:

$$
\begin{aligned}
& N y=\frac{E t}{1-v^{2}}\left(\frac{\partial^{*} v(x, y)}{\partial y}+v \frac{\partial u^{*}(x, y)}{\partial x}\right) \\
& N x y=\frac{E t}{2(1+v)}\left(\frac{\partial u^{*}(x, y)}{\partial y}+\frac{\partial v^{*}(x, y)}{\partial x}\right)
\end{aligned}
$$




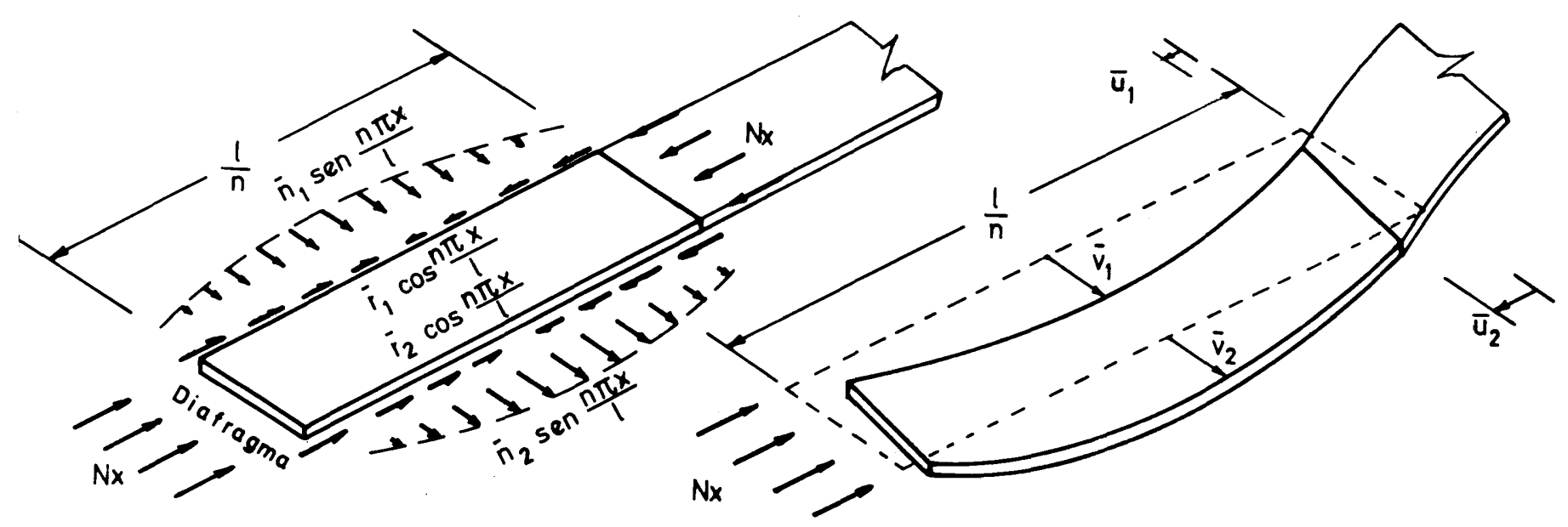

Figura 6

En este caso, los corrimientos en el plano medio de la chapa $u^{*}(x, y)$ y $v^{*}(x, y)$ vienen definidos por las expresiones $1-5,6$, y los coeficientes indeterminados $\mathrm{F}_{n}, \mathrm{G}_{n}, \mathrm{H}_{n}$ y $\mathrm{M}_{n}$, de dichas expresiones, se pueden evaluar imponiendo las condiciones de contorno en los dos bordes longitudinales:

$$
\begin{aligned}
& \text { para } y=0 \quad\left\{\begin{array}{l}
u^{*}(x, y)=\bar{u}_{1} \cos \frac{n \pi x}{1} \\
v^{*}(x, y)=\bar{v}_{1} \operatorname{sen} \frac{n \pi x}{1}
\end{array}\right. \\
& \text { para } y=a\left\{\begin{array}{l}
u^{*}(x, y)=\bar{u}_{2} \cos \frac{n \pi x}{1} \\
v^{*}(x y)=\bar{v}_{2} \operatorname{sen} \frac{n \pi x}{1}
\end{array}\right.
\end{aligned}
$$

Ordenando matricialmente las expresiones II-3, podemos escribir:

$$
\left[\begin{array}{l}
\bar{n}_{1} \\
\bar{r}_{1} \\
\bar{n}_{2} \\
\bar{r}_{2}
\end{array}\right]=\left[\begin{array}{llll}
l_{1,1} & l_{1,2} & l_{1,3} & l_{1,4} \\
l_{2,1} & l_{2,2} & l_{2,3} & l_{2,4} \\
l_{3,1} & l_{3,2} & l_{3,3} & l_{3,4} \\
l_{4,1} & l_{4,2} & l_{4,3} & l_{4,4}
\end{array}\right]\left[\begin{array}{l}
\bar{v}_{1} \\
\bar{u}_{1} \\
\bar{v}_{2} \\
\bar{u}_{2}
\end{array}\right]
$$

cuyos coeficientes $I_{i, j}(i, j=1,2,3$ y 4$)$ hemos tabulado en el apéndice 3 . Estos coeficientes son funciones trascendentes de la solicitación, de la geometría de la chapa y del número entero $n(n=1,2,3, \ldots)$.

c) Comportamiento global.

La matriz de rigidez de la chapa, en la que se considera, de forma simultánea, su comportamiento como placa y como laja, se obtiene ensamblando las expresiones II-2 y 4 . El resultado final es: 


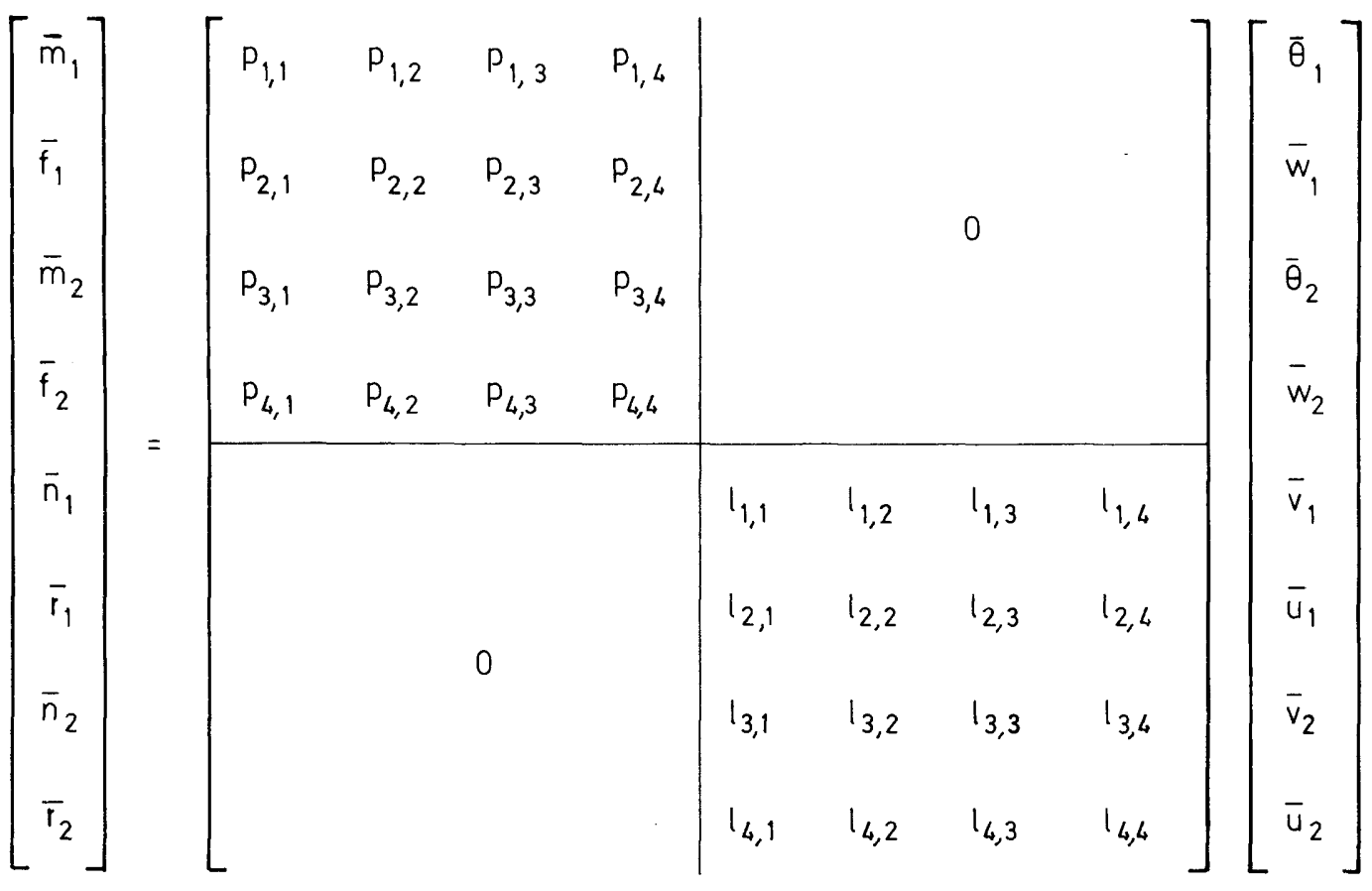

Conviene recordar que la técnica de análisis que hemos aplicado, para deducir la matriz de rigidez de la chapa, es similar a la que se aplica en el método armónico de análisis de láminas plegadai. La única diferencia estriba en que en este trabajo se considera là influencia que tiene la solicitación longitudinal $N_{*}$ en la rigidez de la chapa, que si está traccionada, es más rígida y menos deformable, mientras que, si está comprimida, es menos rígida y más deformable.

\section{II.2. Proceso de formación de la matriz de rigidez del panel rigidizado}

Acabamos de deducir la expresión matricial (matriz de rigidez de la chapa) que relaciona las cargas actuantes en los bordes de la chapa con los movimientos de dichos bordes. Esta relación se ha deducido en un sistema de coordenadas propio de la chapa (coordenadas locales), que se ha seleccionado procurando que las relaciones anteriores tengan una expresión sencilla.

A continuación, debemos hacer un cambio de ejes para expresar estas relaciones en un sistema de coordenadas único que se asocia a toda la estructura (coordenadas globales).

El paso siguiente es ensamblar todas las chapas en las líneas nodales del panel rigidizado. Para ello, debemos establecer en todas las líneas nodales, primero, las ecuaciones de compatibilidad entre los movimientos, es decir, los movimientos de los bordes de las chapas deben ser compatibles con los movimientos de las líneas nodales a los que están unidas, $y$, después, se establecen las ecuaciones de equilibrio entre las cargas, es decir, la suma de las cargas que actúan en los bordes de las chapas que concurren en una línea nodal debe ser igual a la carga exterior actuante sobre dicha línea nodal.

De esta forma, conseguimos relacionar, mediante un conjunto de sistemas de ecuaciones, los movimientos de las líneas nodales con las cargas exteriores aplicadas en dichas líneas nodales.

$\underline{\mathrm{F}_{n}}=\underline{\underline{\mathrm{K}_{n}}} \underline{\underline{\mathrm{D}_{n}}}(\mathrm{n}=1,2,3, \ldots)$

donde:

$\underline{\mathrm{F}_{n}} \quad=$ vector que define las amplitudes de las cargas sinusoidales aplicadas en las líneas nodales.

$\underline{\mathbf{k}_{n}} \quad=$ matriz de rigidez del panel rigidizado.

$\underline{D}_{n} \quad=$ vector que define las amplitudes de los movimientos sinusoidales de las líneas nodales. 
Como no hay cargas exteriores aplicadas en las líneas nodales, ya que la única solicitación es la acción longitudinal, los sistemas anteriores son sistemas homogéneos:

$$
\underline{0}=\underline{\underline{K_{n}}} \underline{\underline{D_{n}}}(\mathrm{n}=1,2,3, \ldots)
$$

siendo sus soluciones no triviales las que definen las cargas críticas y sus modos de pandeo asociados.

Así, pues, para determinar las cargas críticas del panel rigidizado, debemos evaluar para cada $\mathrm{n}$ (número de semiondas longitudinales en el momento del pandeo) los valores de $\lambda=\lambda_{c}$ que anulan el determinante de su matriz de rigidez.

Recordemos que los términos de la matriz de rigidez son funciones trascendentes de $\lambda$ y $\mathrm{n}$, de forma que, en general, para cada valor de $\mathrm{n}$ obtendremos infinitos valores de $\lambda$ que anulan el determinante de $K_{1 . .}$ En realidad sólo nos interesa el menor de todos los valores críticos de $\lambda$.

Dado que los términos de la matriz de rigidez varían con $\lambda$ de una forma compleja, anulándose o haciéndose infinitos en intervalos irregulares, es necesario emplear una técnica iterativa adecuada que garantice la convergencia hacia el menor autovalor. Para ello, se recomienda emplear un algoritmo propuesto inicialmente por Lord Rayleigh, para analizar problemas de dinámica estructural, y que ha sido reelaborado posteriormente por Wittrick (referencia 12), para aplicarlo a problemas de inestabilidad.

\section{II.3. Análisis en ordenador}

Aplicando los criterios que acabamos de exponer hemos preparado un programa de ordenador que permite determinar la menor carga crítica de un panel rigidizado. Los datos de partida son: la geometría de la sección transversal, y la solicitación longitudinal primaria de cada una de las chapas que componen el panel. La discretización del panel debe hacerse de forma que pueda suponerse aproximadamente constante la solicitación longitudinal $\mathrm{N}_{x}$ en cada una de sus chapas componentes.

El programa desarrolla un doble proceso iterativo, ya que, para cada valor de la longitud total del panel, el ordenador debe calcular la menor de las cargas críticas $\lambda_{c}$ correspondientes a los supuestos de que el panel pandee con $n$ semiondas longitudinales (donde $n=1,2,3, \ldots$ ). Este análisis permite construir un gráfico con el que se estudia la evolución de la menor carga crítica en función de la longitud del panel rigidizado.

A modo de ejemplo, en la figura 7 , hemos representado los resultados que se obtienen al analizar el panel de la figura 7a que se ha discretizado descomponiéndolo en 17 chapas ensambladas entre sí en 14 líneas nodales.

Del examen de dicha figura se desprende que, para longitudes del panel inferiores a $6,8 \mathrm{~b}(4,1$ metros), la primera carga crítica se corresponde con un modo de pandeo de abolladura en el que el número de semiondas que se forman, en dirección longitudinal, depende de la longitud de la pieza. El valor de la carga crítica de abolladura es variable, pero una buena aproximación, que nos dejaría del lado de la seguridad, sería suponer que, para longitudes inferiores a 6,8b $\left(4,1\right.$ metros), la menor carga crítica es: $\sigma_{c r}=3.050 \mathrm{~kg} / \mathrm{cm}^{2}$, que se corresponde con el mínimo de todas las ondas que aparecen en el tramo horizontal del diagräma.

Por último, cuando la longitud del panel es superior a $6,8 \mathrm{~b}(4,1$ metros $)$, la primera carga crítica se corresponde con un modo de pandeo por flexión de los rigidizadores, disminuyendo el valor de la menor carga crítica a medida que aumenta la longitud del panel.

De dicho gráfico puede extraerse la conclusión práctica de que, para longitudes grandes del panel, si deseamos mantener la capacidad resistente del mismo dentro de unos márgenes económicos, debemos disponer rigidizadores transversales (diafragmas) con una separación de 4,1 metros. Esta separación es, precisamente, la longitud del panel para la que coincide el modo de pandeo por abolladura con un modo de pandeo por flexión generalizada. Disponer rigidizadores transversales con una separación menor a la apuntada anteriormente no tiene ninguna utilidad práctica. 


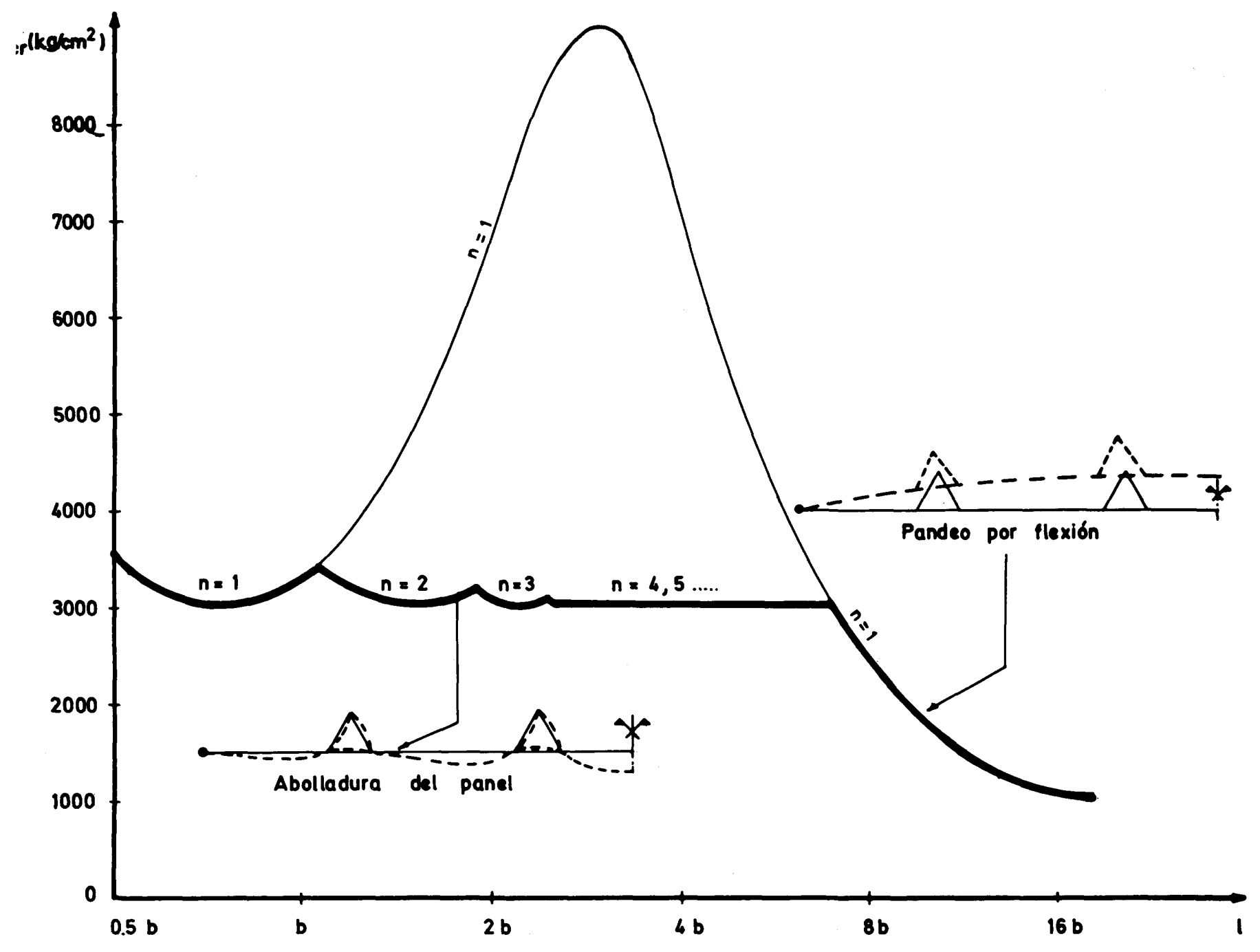

Figura 7
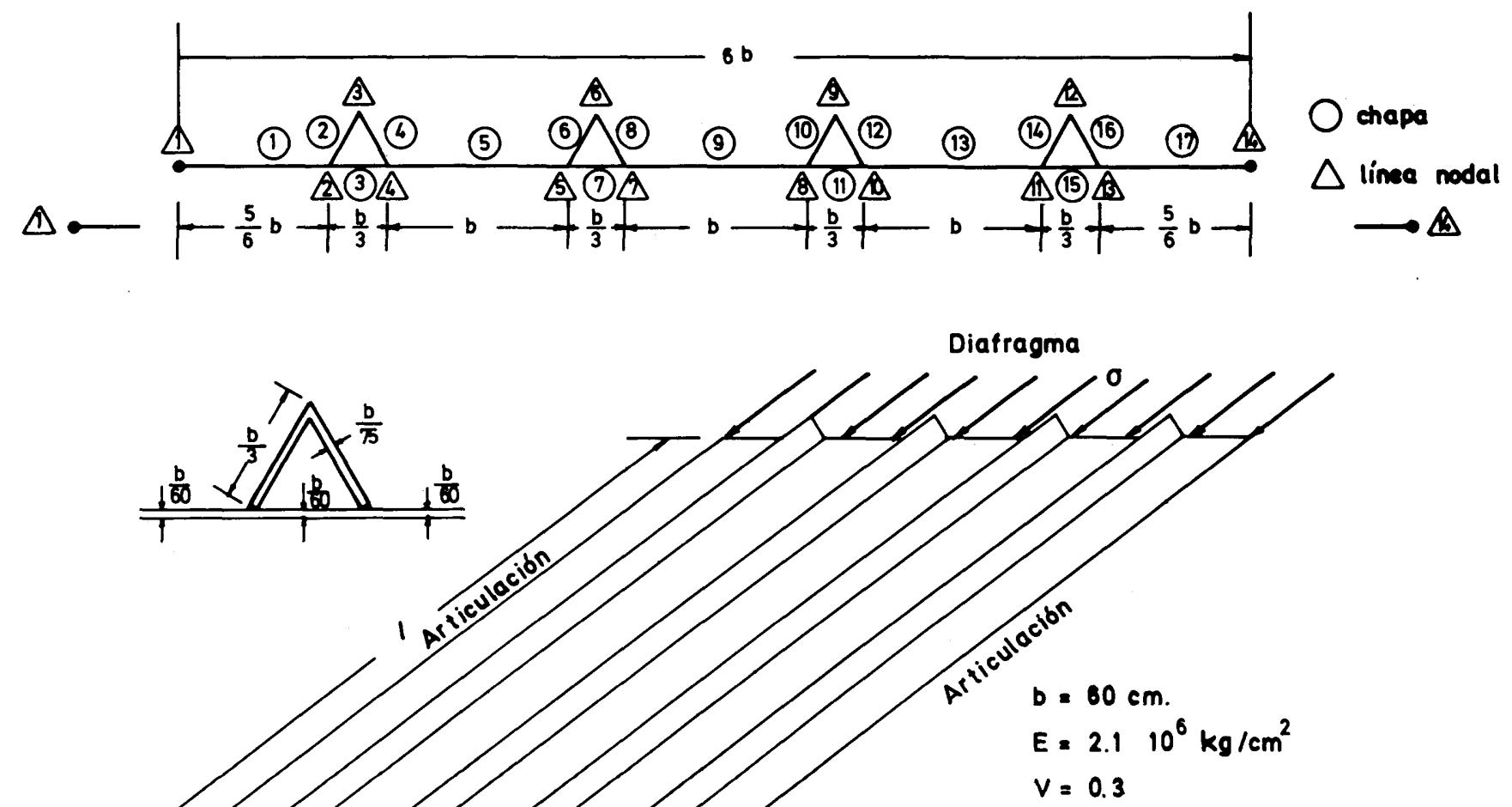

Valor de "l" varia entre $0.5 \mathrm{~b}$ y $20 \mathrm{~b}$ 


\section{Tensiones residuales longitudinales inducidas en el proceso de soldadura de los rigidizadores}

Durante la soldadura al arco eléctrico de los rigidizadores longitudinales del panel, se produce un calentamiento intenso en la zona en la que se va depositando el cordón. La zona calentada, que se encuentra en estado plástico, intenta dilatarse, produciéndose un recalcado del material de esta zona al coartar su dilatación el material de las proximidades que se encuentra frío. Posteriormente, cuando el material calentado vuelve a enfriarse, se producen contracciones longitudinales que, al ser coartadas por el material colindante, inducen tensiones residuales.

El análisis teórico de las tensiones residuales inducidas durante la soldadura es bastante complejo. Por esta causa, la mayoría de los estudios realizados son de tipo experimental. En la figura 8 hemos representando, como ejemplo, la distribución de tensiones residuales longitudinales medida por Fukumoto $(7,8)$ en un panel de tres rigidizadores abiertos.

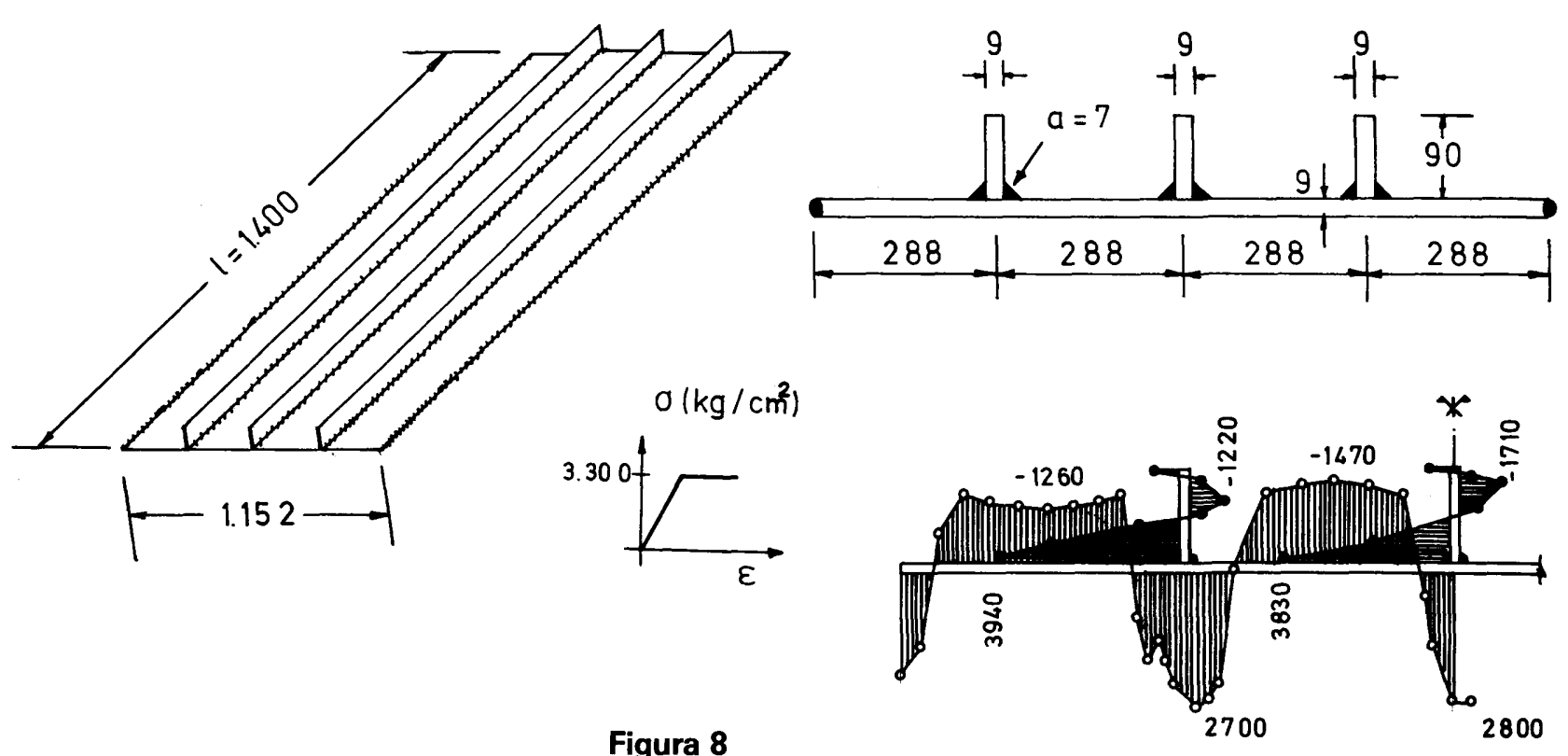

Actualmente, se dispone de criterios simplificados que permiten estimar el valor de dichas tensiones residuales en función de la geometría de la sección transversal del panel y del proceso de soldadura aplicado. Los modelos de distribución que suelen emplearse con más frecuencia son los indicados en la figura 9.

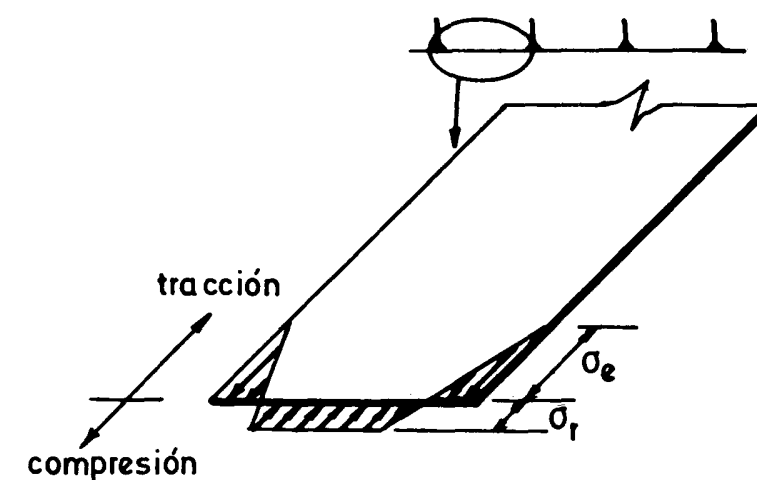

compresión

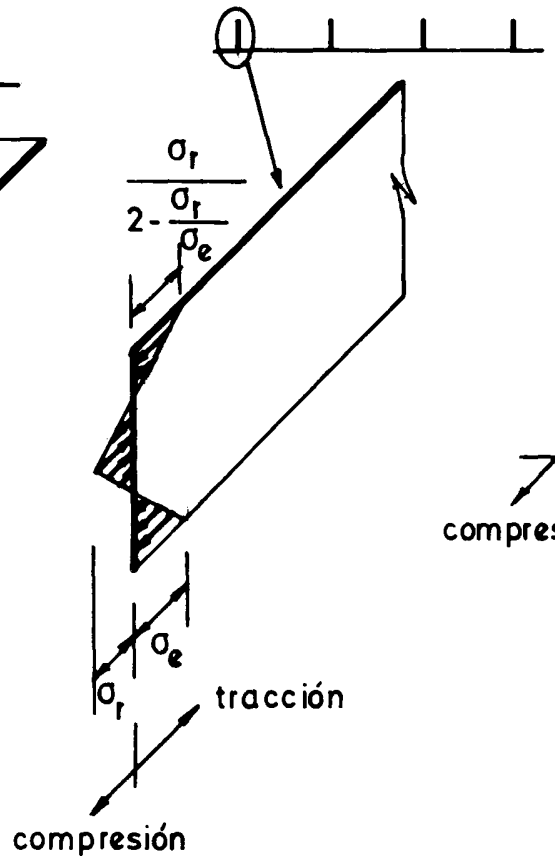

Figura 9 
Estas tensiones residuales, que constituyen un sistema de tensiones autoequilibrado, quedan en el panel y tendrán una marcada influencia en el comportamiento resistente del mismo, especialmente, frente a problemas de inestabilidad.

Para estudiar la interacción entre las tensiones residuales y las tensiones inducidas por las cargas exteriores, en la figura 10 representamos el comportamiento en tensiones y deformaciones de una de las chapas que componen el panel, para valores crecientes de la solicitación longitudinal.

DEFORMACIONES
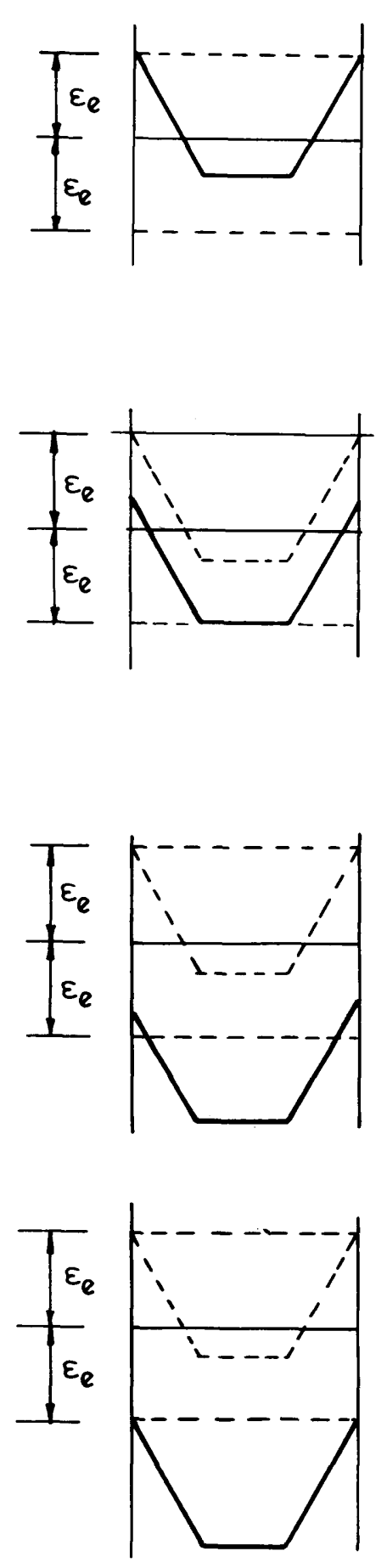

\section{TENS IONES}

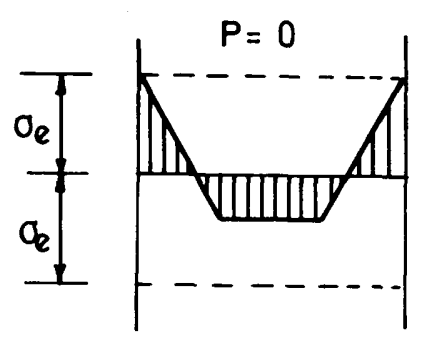

$P=A\left(\sigma_{e}-\sigma_{r}\right)$

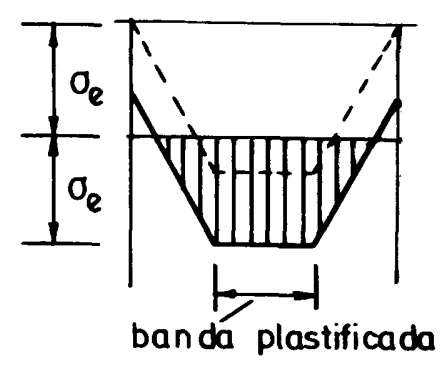

ban da plastificada
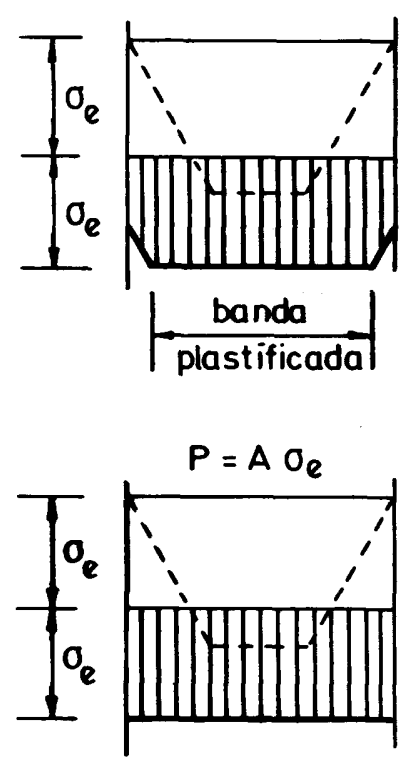

está plastificada
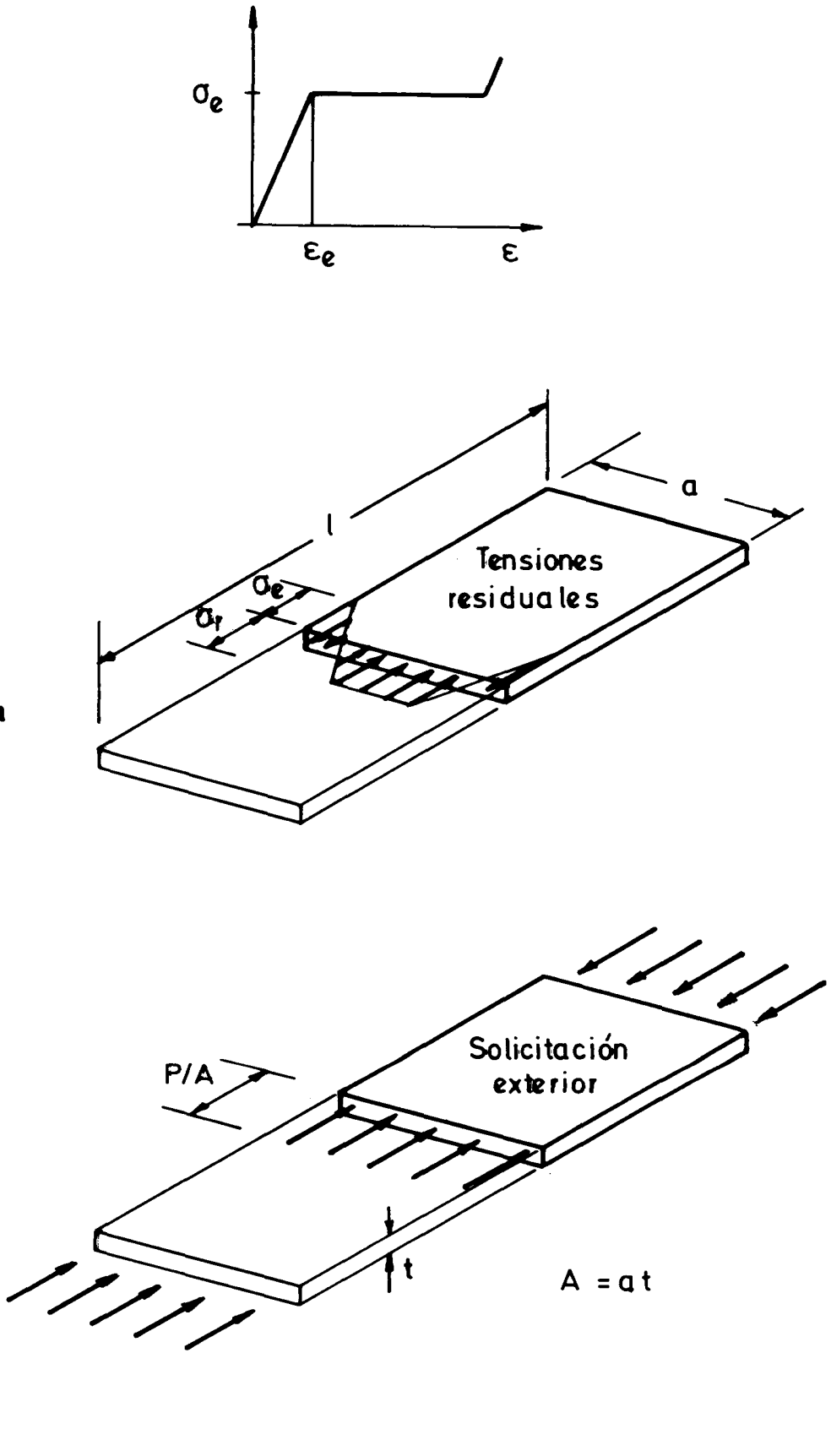

Figura 10 
Puede observarse en estos gráficos que la chapa de acero se comporta en régimen elástico siempre que la solicitación longitudinal no alcance el valor: $P=A\left(\sigma_{e}-\sigma_{r}\right)$. Cuando se alcanza dicha solicitación, se inicia la plastificación en la zona central, plastificación que va progresando hacia los bordes a medida que crece la solicitación. Finalmente, decimos que la chapa agota su capacidad resistente cuando la carga aplicada alcanza el valor: $\mathrm{P}=\mathrm{A} \sigma_{e}$ ya que, en ese momento, toda la chapa está plastificada.

Cuando el pandeo del panel se produce antes de que se inicien las plastificaciones, decimos que el panel pandea en régimen elástico, mientras que, si el pandeo se produce después de haberse iniciado las plastificaciones de ciertas zonas de sus chapas componentes, entonces decimos que el panel pandea en régimen elastoplástico.

\section{Influencia de las tensiones residuales de soldadura en el pandeo en régimen elástico de paneles rigidizados}

Modificando ligeramente la técnica matricial de análisis armónico, que hemos desarrollado en el apartado II, podemos considerar la influencia que tienen las tensiones residuales de soldadura en el pandeo en régimen elástico de paneles metálicos rigidizados.

Para ello, basta con analizar el problema, teniendo en cuenta que el esfuerzo primario que solicita al panel rigidizado viene definido por:

$$
\mathrm{N}_{x}=\mathrm{N}_{x_{r}}+\lambda \mathrm{N}_{x_{0}}
$$

donde:

$\mathrm{N}_{x}=$ esfuerzo primario que solicita el panel rigidizado.

$N_{x}$ = esfuerzo residual inducido en el proceso de soldadura de sus rigidizadores.

$N_{x_{0}}=$ esfuerzo inducido en el panel por las cargas exteriores cuando el parámetro de carga $\lambda$ vale la unidad.

$\lambda=$ parámetro de carga que al crecer aumenta la solicitación de las chapas que componen el panel.

El objetivo del análisis es, precisamente, evaluar el menor valor de $\lambda=\lambda_{c 1}$ a partir del cual el sistema estructural deja de estar en equilibrio estable en la llamada geometría inicial. Como hemos visto en el apartado II, esto supone buscar el menor valor de $\lambda$ que anula el determinante de los coeficientes de los sistemas homogéneos:

$$
\underline{0}=\underline{\underline{K_{n}}} \mathrm{D}_{n} \text { para } \mathrm{n}=1,2,3, \ldots
$$

Debemos recordar que el método armónico que venimos exponiendo discretiza el panel en chapas rectangulares, de forma que la solicitación longitudinal de cada una de estas chapas se pueda suponer aproximadamente constante en dirección transversal.

Como las tensiones residuales tienen una variación muy pronunciada en dirección transversal nos veremos obligados, si deseamos obtener resultados precisos, a discretizar cada chapa, descomponiéndola en varias chapas de anchura reducida e introduciendo varias líneas nodales en la zona de variación de dichas tensiones residuales.

Esta forma de operar tiene el inconveniente de que aumenta de forma notable el número de grados de libertad del sistema estructural $y$, por consiguiente, aumenta la dificultad numérica del proceso de cálculo.

No obstante, el problema puede simplificarse, ya que normaimente todos los rigidizadores son iguales, tienen la misma distribución de tensiones residuales y, además, se disponen con separación uniforme.

Así, pues, podemos analizar, en primer lugar, los rigidizadores y las porciones de chapa que los unen, deduciendo sus matrices de rigidez condensadas, en las que se eliminan los grados de libertad de movimiento de todas las líneas nodales que hemos tenido que introducir en puntos intermedios. Posteriormente, ensamblando estas matrices de rigidez condensadas, generamos la matriz de rigidez del sistema estructural. 
De esta forma, la matriz de rigidez del panel tiene el mismo tamaño que la obtenida en los análisis en que no incluíamos la influencia de las tensiones residuales.

Esta idea se puede generalizar analizando por separado, no sólo una de las chapas que componen el panel, sino un conjunto de chapas que formen una subestructura que se repita regularmente. De este modo, conseguimos una notable economía de cálculo (figura 11).

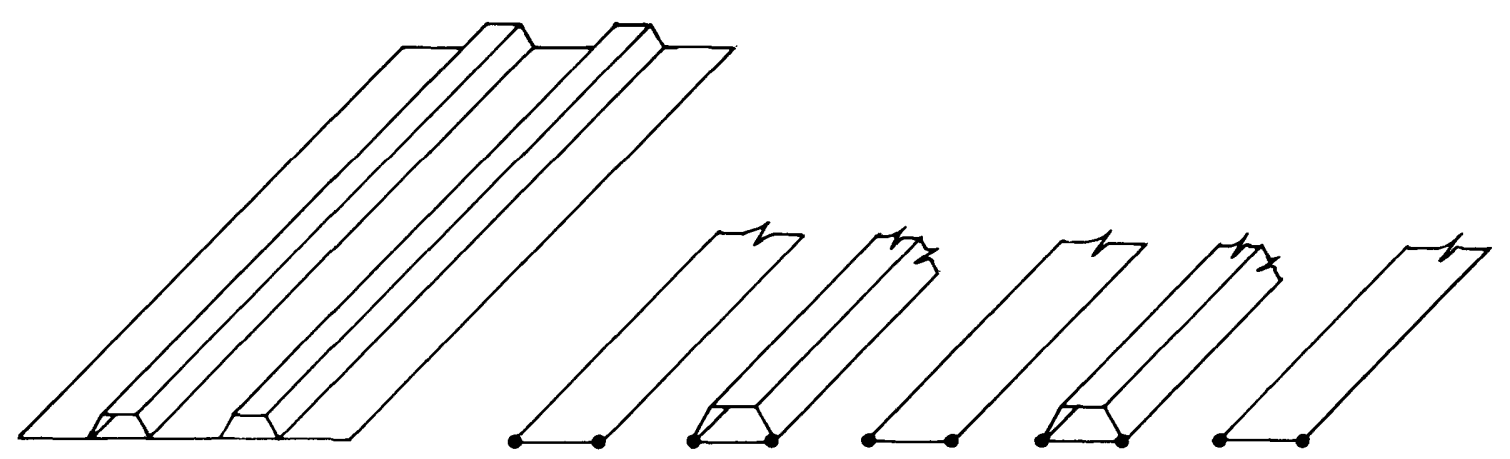

Figura 11

Aplicando los criterios que acabamos de exponer, hemos preparado un programa de ordenador que permite estudiar la influencia que tienen las tensiones residuales de soldadura en el valor de la menor carga crítica de un panel rigidizado, en el supuesto de que su pandeo se produzca en régimen elástico.

Los datos de partida del programa son: la geometría de la sección transversal del panel, la solicitación longitudinal, la distribución de tensiones residuales de soldadura en cada una de las chapas que componen el panel y el número de bandas longitudinales en que se desea discretizar las zonas de las chapas en que existe variación transversal de la solicitación longitudinal.

Con este programa hemos procesado el panel de la figura 12a. El análisis lo hemos hecho para tres valores diferentes de las tensiones residuales de soldadura, los resultados que se han obtenido, para diversos valores de la longitud del panel, los representamos en la figura 12.

Del examen de dicha figura se desprende que, cuando el pandeo del panel es por flexión de los rigidizadores, las tensiones residuales no influyen en el comportamiento del panel. Sin embargo, cuando el pandeo del panel es por abolladura de sus chapas, las tensiones residuales provocan una disminución en el valor de la menor carga crítica del panel. Esta disminución es tanto más importante a medida que aumenta el valor de las tensiones residuales de soldadura.

\section{Influencia de las tensiones residuales de soldadura en el pandeo en régimen elastoplástico de paneles rigidizados}

Para que el pandeo del panel rigidizado tenga lugar en régimen elástico, es necesario que, en el momento del pandeo, la tensión de comparación en todos los puntos del panel se mantenga inferior al límite elástico del material estructural. Esto se dará únicamente en paneles muy esbeltos y, en consecuencia, poco económicos, ya que está desaprovechada la capacidad resistente del acero.

Normalmente, cuando se produce el pandeo en los paneles reales nos encontramos con unas bandas longitudinales que se encuentran plastificadas, mientras que otras bandas longitudinales están todavía en régimen elástico (figura 10). 


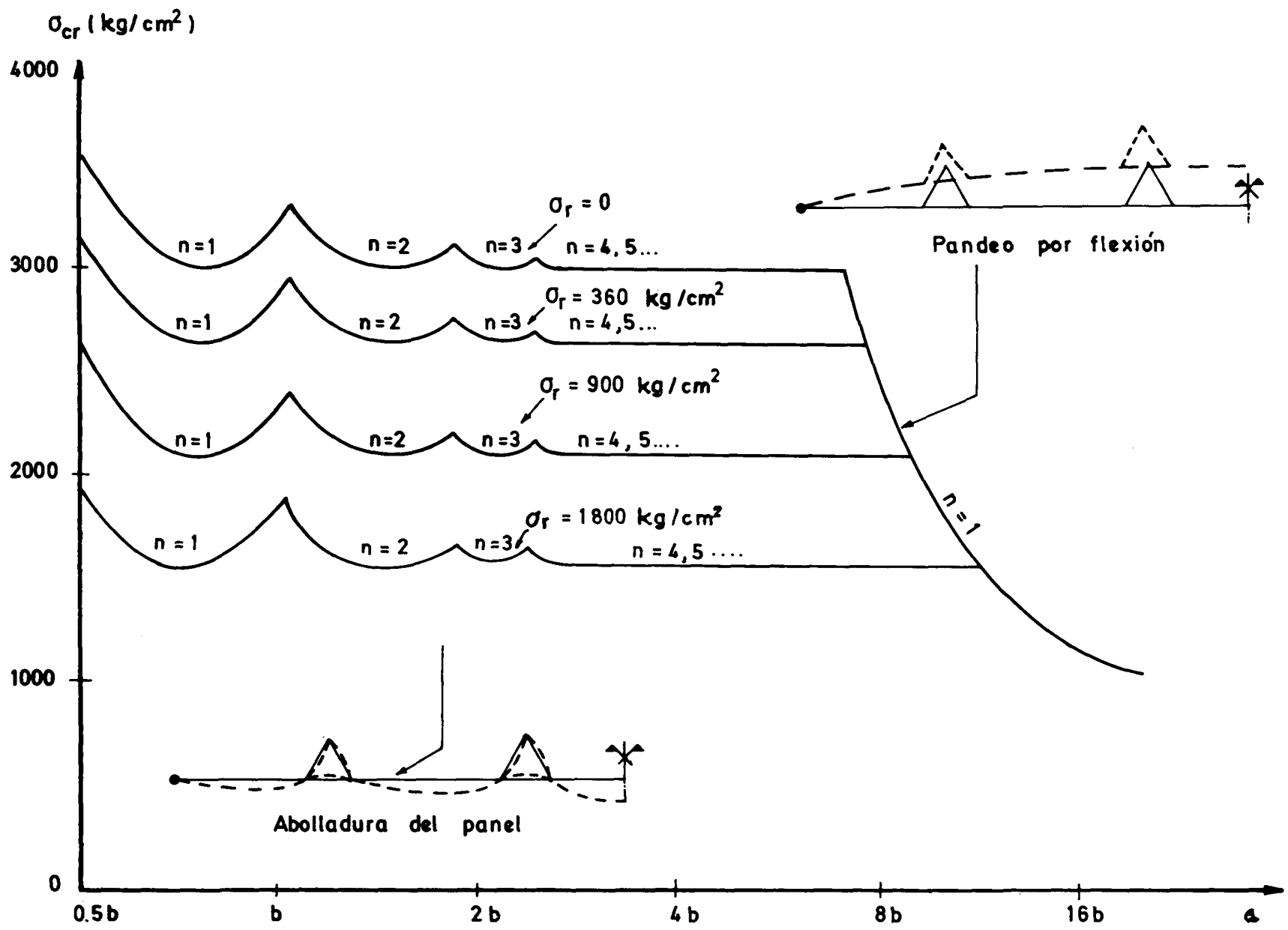

Figura 12

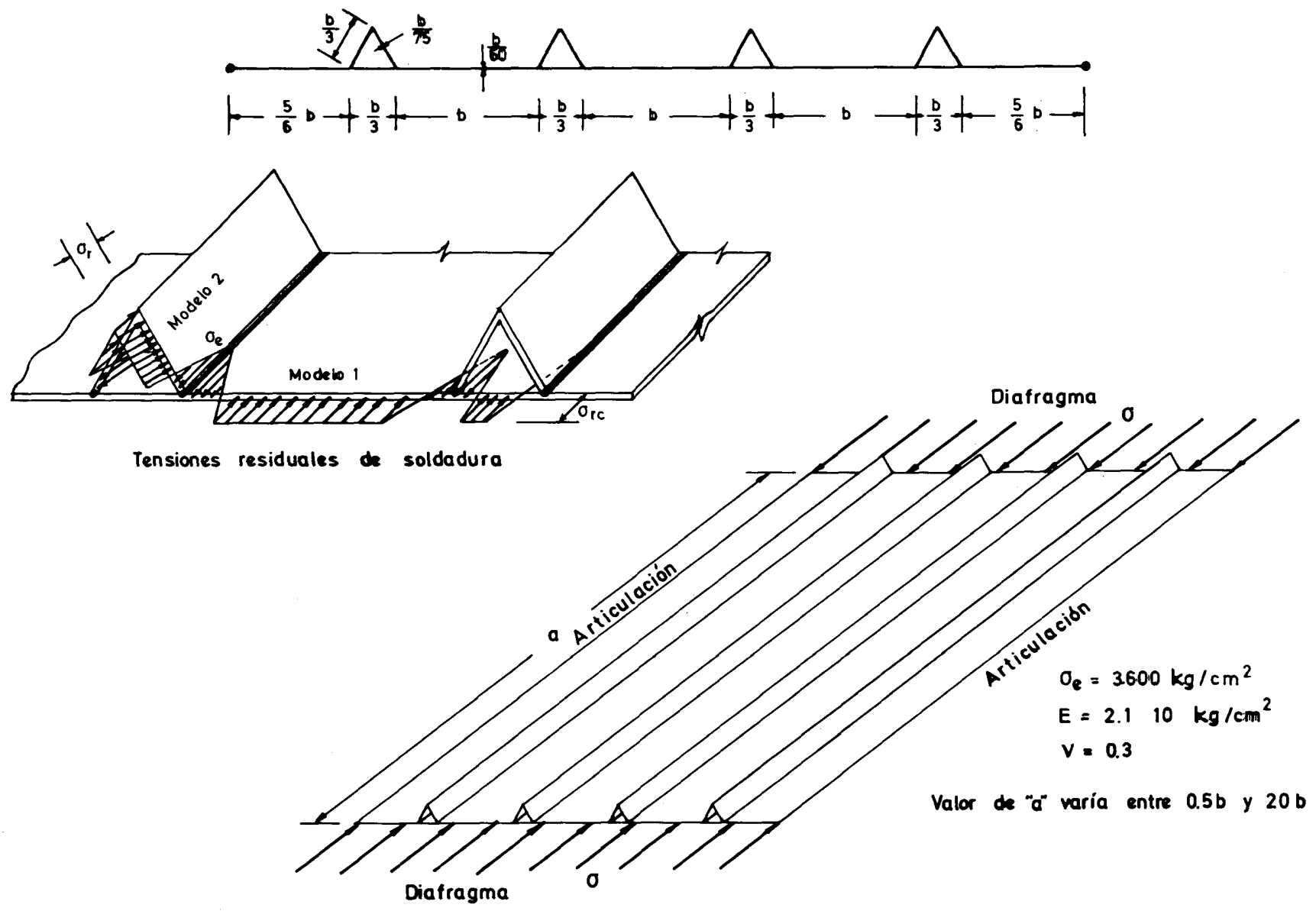

Figura 12a 
El método matricial de análisis armónico, que venimos desarrollando, puede generalizarse de forma que también permita analizar el pandeo en régimen elastoplástico de paneles rigidizados. Lo único que nos falta por definir es el comportamiento, en el instante del pandeo, de las bandas longitudinales que se encuentran plastificadas.

\section{V.1. Comportamiento, en el instante del pandeo, de una chapa metálica que se encuentra plastificada}

Empezaremos analizando una banda longitudinal que se encuentra solicitada por una tensión de compresión de valor $\sigma_{e}$, en el supuesto de que su deformación longitudinal venga definida por el punto $\mathrm{M}$ del diagrama $\sigma-\varepsilon$.
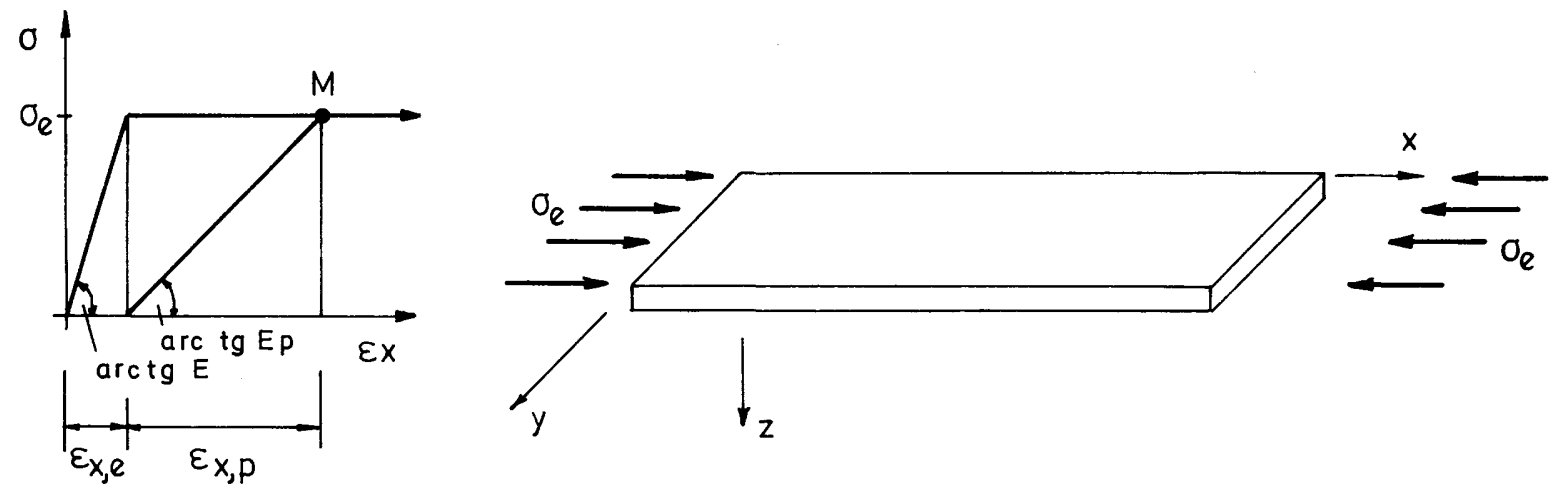

Figura 13

Recordemos que en el instante del pandeo de la chapa, tanto si pandea con un comportamiento tipo laja, como si lo hace con un comportamiento tipo placa, la chapa adopta una geometría ligeramente deformada, muy próxima a la geometría inicial. Por consiguiente, se generan unas tensiones y deformaciones infinitesimales que se superponen con las inducidas por la solicitación primaria existente antes de producirse el pandeo.

El primer problema que debemos abordar es la deducción de las expresiones que relacionan entre sí las tensiones y deformaciones infinitesimales que se producen en el momento del pandeo. Existen varias teorías de pandeo plástico que permiten evaluar dichas relaciones (Bleich, Kaufmann, Bijlaard, llyushin, Stowell, Handelman y Prager, etc.). De todas ellas hemos seleccionado la de Bijlaard (13), por ser la que viene utilizándose en los últimos trabajos que se han publicado sobre el pandeo elastoplástico de paneles rigidizados (Fukumoto - Usami, Hasegawa - Ota, etc.).

La teoría del pandeo plástico de Bijlaard se basa en tres hipótesis simplificadoras:

a) En primer lugar, se admite como criterio de plastificación el de Huber, Hencky y von Mises.

b) En segundo lugar, se admite que, en el momento del pandeo, la solicitación primaria aumenta ligeramente, de forma que la tensión de comparación se mantiene igual al límite elástico en todos los puntos de la chapa. En consecuencia no se producen procesos de descarga del material. Esta hipótesis es una extrapolación de la teoría del módulo tangente de Engesser y $\mathrm{V}$. Karman, que es una generalización excelente del concepto «carga crítica» al campo plástico.

c) Por último, se supone que la parte plástica de las deformaciones totales admite un tratamiento similar al de la parte elástica de dichas deformaciones. Las únicas diferencias radican en que, en el dominio plástico, el coeficiente de Poisson pasa a valer $1 / 2$, ya que las deformacionés plásticas tienen lugar sin cambio de volumen. Además el módulo de elasticidad $E$ hay que sustituirlo por un módulo secante $\mathrm{E}_{p}$ (figura 13). 
Concretando más, podemos escribir:

$$
\begin{array}{rlrl}
\varepsilon_{x} & =\varepsilon_{x, e}+\varepsilon_{x, p} & \\
\varepsilon_{y} & =\varepsilon_{y, e}+\varepsilon_{y, p} & \\
\gamma_{x y} & =\gamma_{x y, e}+\gamma_{x y, p} & \\
\text { donde: } \quad \varepsilon_{x, e} & =\frac{1}{E}\left(\sigma_{x}-v \sigma_{y}\right) & \varepsilon_{x, p} & =\frac{1}{E p}\left(\sigma_{x}-\frac{1}{2} \sigma_{y}\right) \\
\varepsilon_{y, e} & =\frac{1}{E}\left(\sigma_{y}-v \sigma_{x}\right) & \varepsilon_{y, p} & =\frac{1}{E p}\left(\sigma_{y}-\frac{1}{2} q_{x}\right) \\
\gamma_{x y, e} & =\frac{2(1+v)}{E} \tau_{x y} & \gamma_{x y, p} & =\frac{3}{E p} \tau_{x y}
\end{array}
$$

partiendo de estas hipótesis simplificadoras, se puede demostrar (ver la referencia 13) que la relación existente entre las tensiones y deformaciones infinitesimales, que se inducen cuando la chapa pandea en régimen plástico, vienen definidas por:

$$
\begin{aligned}
& \sigma_{x}^{*}=\frac{E}{3 \frac{E}{E p}+5-4 v}\left(\varepsilon_{x}^{*}+2 \varepsilon_{y}^{*}\right) \\
& \sigma_{y}^{*}=\frac{E}{3 \frac{E}{E p}+5-4 v}\left(\varepsilon_{x}^{*}+2 \varepsilon_{y}^{*}\right) \\
& \tau_{x y}^{*}=\frac{E}{3 \frac{E}{E p}+2+2 v} \gamma_{x y}^{*}
\end{aligned}
$$

Por consiguiente, los esfuerzos secundarios, que se inducen en el momento del pandeo, vienen definidos por las expresiones:

a) Comportamiento placa.

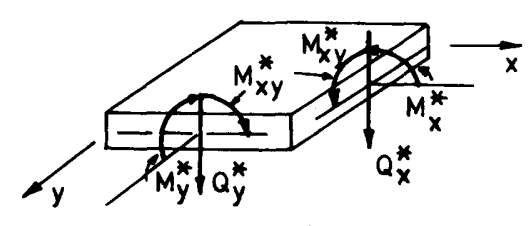

Figura 14

$$
\begin{aligned}
& M_{x}^{*}=-R\left(\frac{\partial^{2} x^{*}(x, y)}{\partial x^{2}}+2 \frac{\partial^{2} w^{*}(x, y)}{\partial y^{2}}\right) \\
& M_{y}^{*}=-2 R\left(\frac{\partial^{2} w^{*}(x, y)}{\partial x^{2}}+2 \frac{\partial^{2} w^{*}(x, y)}{\partial y^{2}}\right) \\
& M_{x}^{*} y=2 T \frac{\partial^{2} w^{*}(x, y)}{\partial x \partial y} \\
& Q_{x}^{*}=-\left(R \frac{\partial^{3} w^{*}(x, y)}{\partial x^{3}}+2(R+T) \frac{\partial^{3} w^{*}(x, y)}{\partial x \partial y^{2}}\right) \\
& Q_{y}^{*}=-\left(4 R \frac{\partial^{3} w^{*}(x, y)}{\partial x^{3}}+2(R+T) \frac{\partial^{3} w^{*}(x, y)}{\partial x^{2} \partial y}\right)
\end{aligned}
$$




$$
R=\frac{E t^{3}}{12\left(3 \frac{E}{E p}+5-4 V\right)}
$$

$$
T=\frac{E t^{3}}{12\left(3 \frac{E}{E p}+2+2 v\right)}
$$

b) Comportamiento laja.

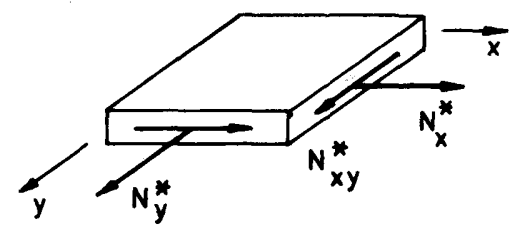

Figura 15

$$
\begin{gathered}
N^{*}=\operatorname{At}\left(\frac{\partial u^{*}(x, y)}{\partial x}+2 \frac{\partial v^{*}(x, y)}{\partial y}\right) \\
N^{*} y=2 A t\left(\frac{\partial u^{*}(x, y)}{\partial x}+2 \frac{\partial v^{*}(x, y)}{\partial y}\right) \\
N^{*} y=B t\left(\frac{\partial u^{*}(x, y)}{\partial y}+2 \frac{\partial v^{*}(x, y)}{\partial x}\right) \\
B=\frac{E}{3 \frac{E}{E p}+2+2 v}
\end{gathered}
$$

Las ecuaciones diferenciales, que establecen el equilibrio de la chapa plastificada en una posición ligeramente deformada, son:

a) Comportamiento placa.

$$
R \frac{\partial^{4} w^{*}}{\partial x^{4}}+4(R+T) \frac{\partial^{4} w^{*}}{\partial x^{2} \partial y^{2}}+4 R \frac{\partial^{4} w^{*}}{\partial y^{4}}-\left( \pm \sigma_{e}\right)+\frac{\partial^{2} w^{*}}{\partial x^{2}}=0
$$

b) Comportamiento laja.

$$
\begin{aligned}
& \left(A \pm \sigma_{e}\right) \frac{\partial^{2} u^{*}}{\partial x^{2}}+B \frac{\partial^{2} u^{*}}{\partial y^{2}}+(2 A+B) \frac{\partial^{2} v^{*}}{\partial x \partial y}=0 \\
& \left(B \pm O_{e}\right) \frac{\partial^{2} v^{*}}{\partial x^{2}}+4 A \frac{\partial^{2} v^{*}}{\partial y^{2}}+(2 A+B) \frac{\partial^{2} u^{*}}{\partial x \partial y}=0
\end{aligned}
$$

el doble signo $\left( \pm \sigma_{e}\right)$ de las expresiones anteriores es para considerar la posibilidad de que la chapa se encuentre traccionada o comprimida.

Una solución, en serie de Levi, de la forma:

$$
\begin{aligned}
& v^{*}(x, y)=\sum_{n=1}^{\infty} w_{n}(y) \operatorname{sen} \frac{n \pi x}{1} \\
& u^{*}(x, y)=\sum_{n=1}^{\infty} u_{n}(y) \cos \frac{n \pi x}{1} \\
& v^{*}(x, y)=\sum_{n=1}^{\infty} v_{n}(y) \operatorname{sen} \frac{n \pi x}{1}
\end{aligned}
$$


satisface las condiciones de contorno en los extremos de la chapa en los que existen diafragmas. Para que $\mathrm{w}^{*}(\mathrm{x}, \mathrm{y})$ verifique la ecuación $\mathrm{V}-1$, la función $\mathrm{W}_{n}(\mathrm{y})$ debe ser:

a) Chapa traccionada.

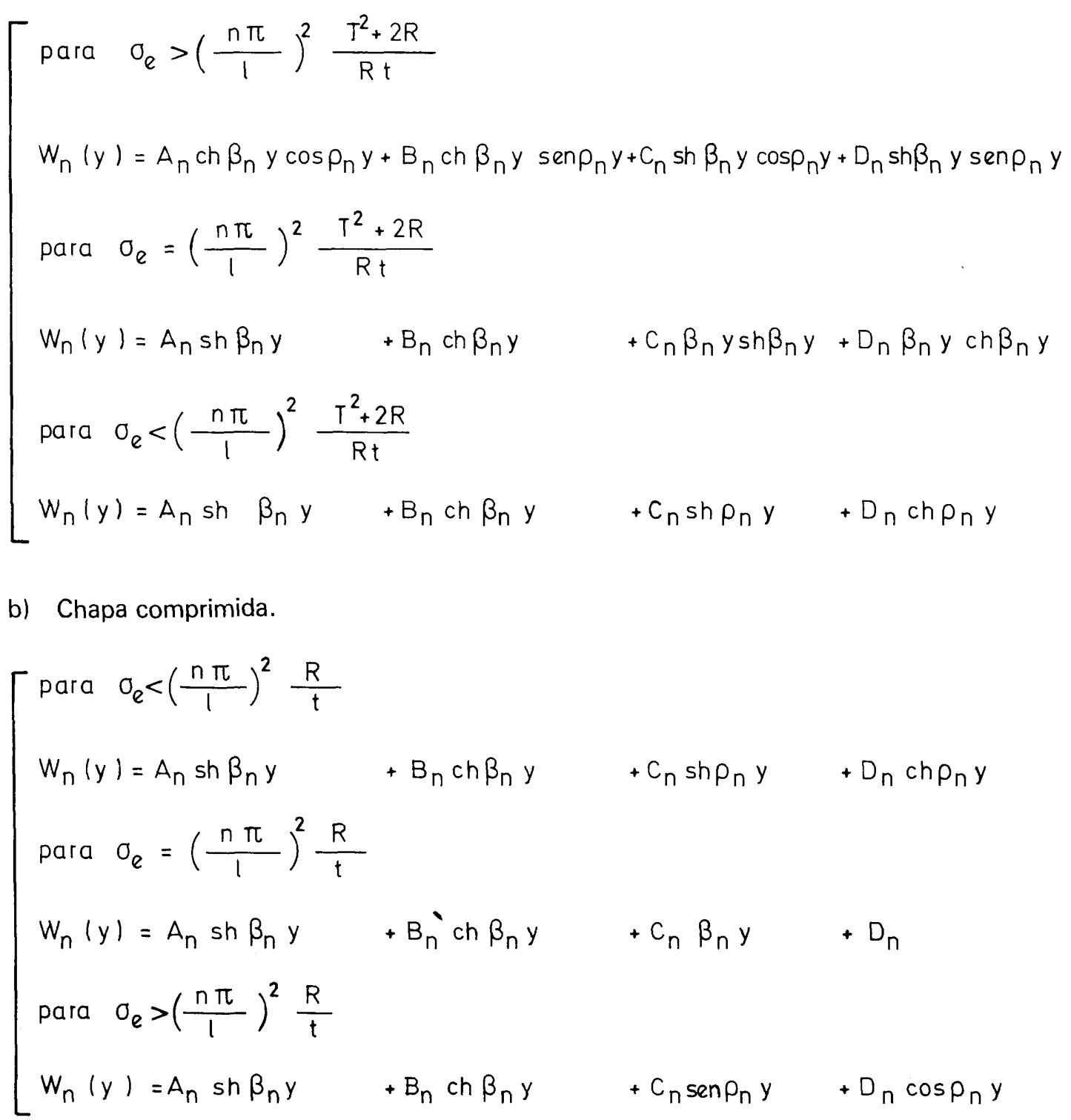

Análogamente, para que $u^{*}(x, y)$ y $v^{*}(x, y)$ verifiquen las ecuaciones $V-2$ y 3 , las funciones $U_{n}(y)$ y $\mathrm{V}_{n}(\mathrm{y})$ deben ser:

\section{a) Chapa traccionada.}

$$
\left[\begin{array}{l}
U_{n}(y)=F_{n} \operatorname{ch} \delta_{n} y \cos Y_{n} y+G_{n} \operatorname{ch} \delta_{n} y \operatorname{sen} \gamma_{n} y+H_{n} \operatorname{sh} \delta_{n} y \cos \gamma_{n} y+M_{n} \operatorname{sh} \delta_{n} y \operatorname{sen} Y_{n} y \\
V_{n}(y)=\left(\delta_{n} f H_{n}-\gamma_{n} g G_{n}\right) \operatorname{ch} \delta_{n} y \cos \gamma_{n} y+\left(\gamma_{n} g F_{n}+\delta_{n} f M_{n}\right) \operatorname{ch} \delta_{n} y \operatorname{sen} \gamma_{n} y+\left(\delta_{n} f F_{n}-\gamma_{n} g M_{n}\right) \\
\operatorname{sh} \delta_{n} y \cos Y_{n} y+\left(\delta_{n} f G_{n}+\gamma_{n} g H_{n}\right) \operatorname{sh} \delta_{n} y \operatorname{sen} \gamma_{n} y \\
f=\frac{\left(A+\sigma_{e}\right)\left(\frac{n \pi}{l}\right)^{2}-B\left(\delta_{n}^{2}+\gamma_{n}^{2}\right)}{\left(\delta_{n}^{2}+Y_{n}^{2}\right)(2 A+B)\left(\frac{n \pi}{1}\right)} \quad g=\frac{\left(A+\sigma_{e}\right)\left(\frac{n \pi}{l}\right)^{2}+B\left(\delta_{n}^{2}+\gamma_{n}^{2}\right)}{\left(\delta_{n}^{2}+\gamma_{n}^{2}\right)(2 A+B)\left(\frac{n \pi}{1}\right)}
\end{array}\right.
$$


b) Chapa comprimida.

$$
\left[\begin{array}{l}
U_{n}(y)=F_{n} \cos \delta_{n} y+G_{n} \operatorname{sen} \delta_{n} y+H_{n} \cos \gamma_{n} y+M_{n} \operatorname{sen} \gamma_{n} y \\
V_{n}(y)=-f G_{n} \cos \delta_{n} y+f F_{n} \operatorname{sen} \delta_{n} y-g M_{n} \cos Y_{n} y+g H_{n} \operatorname{sen} Y_{n} y \\
f=\frac{\delta_{n}^{2} B+\left(\frac{n \pi}{l}\right)^{2}\left(A-\sigma_{e}\right)}{\delta_{n}^{2}\left(\frac{n \pi}{l}\right)(2 A+B)} \quad g=\frac{\gamma_{n} B+\left(\frac{n \pi}{1}\right)^{2}\left(A-\sigma_{e}\right)}{\gamma_{n}\left(\frac{n \pi}{1}\right)(2 A+B)}
\end{array}\right.
$$

Los coeficientes $\beta_{n}, \varrho_{n}, \delta_{n}$ y $\gamma_{n}$ son función de la deformación longitudinal $\varepsilon_{x_{x} p}$, de la geometría de la chapa y del número entero $n(n=1,2,3, \ldots$ ). Los valores de estos coeficientes se han tabulado en el apéndice 2.

\section{V.2. Matriz de rigidez de la chapa plastificada}

Siguiendo un proceso similar al que expusimos en el apartado II, podemos deducir la expresión matricial que, en una chapa plastificada, relaciona un sistema de cargas sinusoidales aplicadas en sus dos bordes con los movimientos sinusoidales que dichas cargas provocan (figuras 5 y 6). Debemos indicar que, en este caso, tanto las cargas como los movimientos tienen un carácter infinitesimal. El resultado que se obtiene es:

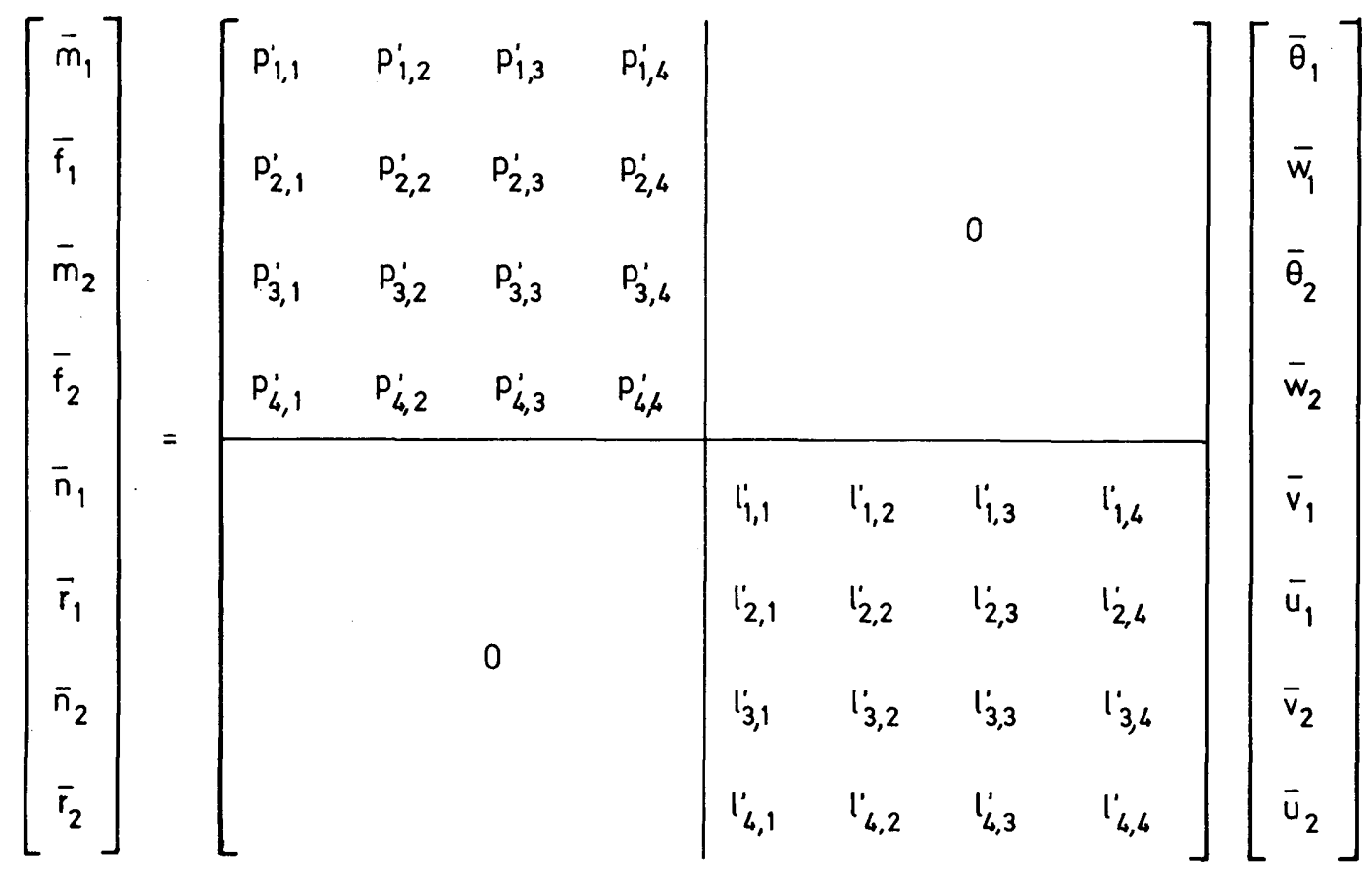

cuyos coeficientes $p_{i j}^{\prime}$ y $l_{i j}^{\prime}(i, j=1,2,3$ y 4$)$ hemos tabulado en el apéndice 4. Estos coeficientes son funciones trascendentes de la deformación longitudinal de la chapa plastificada, de su geometría $y$ del número entero $n(n=1,2,3, \ldots)$.

\section{V.3. Pandeo elastoplástico de paneles rigidizados}

Una vez que hemos deducido las matrices de rigidez de todas las chapas en que hemos discretizado el panel, podemos ensamblarlas entre sí en las líneas nodales, estableciendo las ecuaciones de compatibilidad y equilibrio. 
$\underline{\mathrm{O}}=\underline{\underline{\mathrm{K}_{n}}} \underline{\underline{\mathrm{D}_{n}}}$ con $\mathrm{n}=1,2,3, \ldots$

el objeto de nuestro análisis es determinar, en un proceso de carga creciente, cuándo el sistema anterior admite infinitas soluciones. Para ello, debemos buscar cuándo se anula por primera vez alguno de los determinantes de $\underline{\underline{\mathrm{K}_{n}}}(\mathrm{n}=1,2, \ldots)$.

Debemos recordar nuevamente que el método armónico que venimos exponiendo nos exige discretizar el panel en chapas rectangulares, de forma que en cada una de estas chapas se cumpla la siguiente condición:

a) Si la chapa está en régimen elástico, el esfuerzo primario longitudinal debe ser aproximadamente constante en dirección transversal.

b) Si la chapa está en régimen plástico, la deformación longitudinal debe ser aproximadamente constante en dirección transversal.

Existen zonas del panel en las que los esfuerzos longitudinales o las deformaciones plásticas longitudinales varían en dirección transversal, por consiguiente, debemos hacer una discretización fina de esas zonas, introduciendo líneas nodales intermedias. Por otra parte, como nos interesa conseguir que el número de grados de libertad del sistema estructural no sea demasiado grande $y$, además, estos sistemas estructurales son muy repetitivos, es muy conveniente, como hemos apuntado en el apartado IV, trabajar con subestructuras condensando los grados de libertad, que se han introducido en puntos intermedios, antes de ensamblar las chapas entre sí en las líneas nodales.

Aplicando estos criterios hemos preparado un programa de ordenador que permite determinar la menor carga crítica de un panel rigidizado, en el supuesto de que su pandeo tenga lugar en régimen elastoplástico. Los datos de partida del programa son: la geometría de la sección transversal del panel, la solicitación longitudinal primaria de cada una de sus chapas, la distribución de las tensiones residuales de soldadura, el número de bandas longitudinales en que se desea discretizar las zonas de las chapas en que existe variación transversal de la solicitación longitudinal, y el número de bandas longitudinales en que se desea discretizar la zona plastificada de las chapas en que existe variación transversal de la deformación longitudinal.

Con este programa hemos procesado el panel rigidizado de la figura 16a. Los resultados obtenidos, para diversos valores de la longitud del panel, están representados en la figura 16.

Las curvas 1 y 2 se han obtenido suponiendo que no existen tensiones residuales de soldadura y admitiendo, en el primer caso, un comportamiento elástico y lineal del material estructural, mientras que, en el segundo caso, se ha supuesto un comportamiento elastoplástico del mismo.

Las curvas 3 y 4 se han obtenido suponiendo un comportamiento elastoplástico del material estructural y considerando que existen tensiones residuales de soldadura, de valor: $\stackrel{\sigma_{r}}{-}=0,25$ y ${ }^{\sigma_{r}}=0,50$, respectivamente.

$\sigma_{e}$

Del examen de la figura se desprende que las tensiones residuales, unidas al comportamiento elastoplástico del material estructural, tienen una marcada influencia en el valor de la menor carga crítica de abolladura del panel rigidizado. Por otra parte, también observamos que, cuando el pandeo del panel es por flexión de sus rigidizadores, las tensiones residuales únicamente influyen si la solicitación primaria (carga exterior más tensiones residuales) provoca plastificaciones en los rigidizadores disminuyendo su rigidez a flexión. Este último fenómeno es el que se observa en el tramo $A$ B de la curva número 4.

\section{Conclusión}

En este artículo ha quedado expuesta una técnica matricial, de análisis armónico, que permite analizar el pandeo en régimen elastoplástico de paneles metálicos rigidizados longitudinalmente.

El pandeo, en régimen elástico, de estos sistemas estructurales ha sido estudiado por WittrickWilliams (1, 2), Smith (3, 4), Prezemieniecki (5), Plank-Wittrick (6), etc. Estos autores analizan el panel descomponiéndolo en chapas rectangulares, que suponen ensambladas en un conjunto de líneas 


\section{$\sigma_{\mathrm{cr}}\left(\mathrm{kg} / \mathrm{cm}^{2}\right)$}
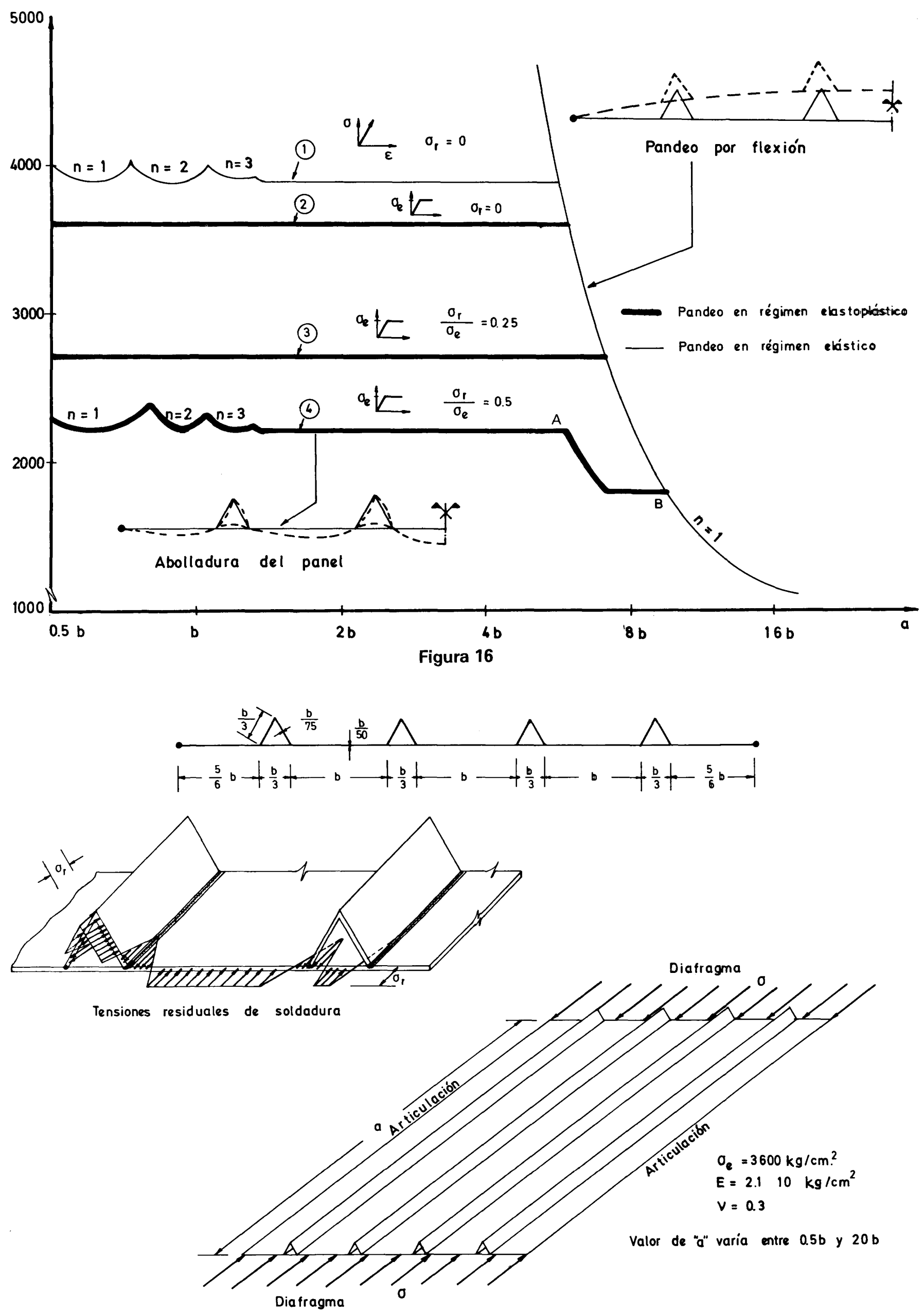

Figura 16a 
nodales. Cada una de estas chapas puede tener un comportamiento tipo placa, con deformadas transversales a su plano medio, o un comportamiento tipo laja, con deformadas contenidas en su plano medio.

Para considerar los esfuerzos de interacción que se desarrollan en las líneas nodales, en las que las chapas se ensamblan entre sí, aplican una técnica matricial de análisis armónico en rigidez. En unos casos, referencias (1) a (4), la matriz de rigidez de cada chapa se deduce integrando directamente las ecuaciones diferenciales que definen su comportamiento (lámina plegada), mientras que en otros casos, referencias (5) y (6), la matriz de rigidez de cada chapa se deduce, suponiendo deformadas aproximadas que se hacen converger hacia la deformada real, aplicando el principio del mínimo de la energia potencial total del sistema (banda finita).

Esta técnica matricial de análisis armónico se puede generalizar, como se ha expuesto en este artículo, de forma que, también, permita analizar el pandeo en régimen elastoplástico de paneles rigidizados. Los autores de este trabajo no conocen ninguna publicación anterior en la que se aborde este problema con toda generalidad. No obstante, debemos indicar, que existen varios trabajos en esa dirección, entre los que cabe citar a Fukumoto-Usami-Okamoto $(7,8)$ y Hasegawa-Otä-Nishino (9). En estos trabajos se aborda el problema de forma parcial, haciendo ciertas simplificaciones, que limitan el análisis al estudio del pandeo elastoplástico de paneles con rigidizadores abiertos de separación uniforme.

NOTA: Este articulo es un resumen de un trabajo de investigación desarrollado, por el segundo de los autores, para la obtención del grado de Doctor.

\section{résumé}

FLAMBEMENT EN REGIME ELASTOPLASTIQUE DE PANNEAUX METAL LIQUES LONGITUDINALEMENT RAIDIS

José Ramón Atienza Reales et Marcos Jesús Pantaleón Prieto, Drs. Ingénieurs des Ponts et Chaussées.

Dans cet article, les auteurs exposent une technique matricielle d'analyse harmonique, qui permet d'analyser le flambement en régime élastoplastique de panneaux raidis sollicités par des charges longitudinales.

Ils rappellent l'existence de deux méthodes de base pour l'étude du flambement d'éléments métalliques comprimés: celle de bifurcation d'équilibre, qui permet de déterminer bifurcation d'équilibre, qui permet de déterminer
les charges CRITIQUES des systèmes les charges CRITIQUES des systèmes PARFAITS, et celle de divergence d'équilibre, SEMENT des systèmes REEtS. La différence essentielle entre les deux méthodes que la première méthode analyse le flambement comme un problème d'équilibre indifférent (infinies solutions) tandis que la seconde analyse le flambement comme un problème de résistance (sommet du diagramme charge-déformation).

Ils analysent le flambement du panneau en le considérant comme étant un problème de bifurcation d'équilibre. Par conséquent, ils supposent que les charges extérieures son parfaitement situés au milieu du panneau et négligent toutes leurs imperfections géométriques.

En premier lieu, ils vont étudier le flambement en régime élastique de ces systèmes structuraux. Ensuite, ils analyseront l'influence que les contraintes résiduelles de la soudure ont sur la valeur de la moindre charge critique, supposé que le flambement ait lieu en régime élastique ou en régime élasto plastique.

\section{summary}

BUCKLING ON ELASTOPLASTIC REGIME OF LONGIUDINALLY RIGIDIZED METAL PANELS

José Ramón Atienza Reales and Marcos Jesús Pantaleón Prieto. Drs. Roads Engineers

In this article we explain a form technique of harmonic analysis, permitting an analysis of buckling on elastoplastic regime of rigidized panels solicited by longitudinal loads.

We call there are two basic methods of studying buckling of compressed metal elements: forking of balance, which permits the CRITICAL loads of the PERFECT systems to be determined, and divergence of balance, permitting the EXHAUSTION loads of the REAL systems to be determined. The basic difference between both methods lies in the fact that the first analyses buckling as an indifferent balance problem (infinite solutions) whereas the second analyses buckling as a problem of resistance (highest point of the load-deformation diagram)

We shall analyse the buckling of the panel, considering it as a balance forking problem. Consequentiy we shall imagine that the outer loads are perfectly centered in the middle of the panel, and we shall discard all their geometric imperfections.

Firstly we are going to study buckling in elastic regime of these structural systems. We shall then go on to analyse the influence the residual welding tensions have at the value of the smaller critical load, in the case of the buckling taking place in elastic regime or in elastoplastic regime.

\section{zusammenfassung}

ELASTOPLASTICHE BEUGUNG LAENGSVERSTAERKTER METALL PLATTEN

José Ramón Atienza Reales und Marcos Jesús Pantaleón Prieto. Drs. Strassenbauingenieure.

In diesem Artikel legen wir eine Gesenk technik mit harmonischer Prüfung dar, die gestattet, die elastoplastische Beugung längsverstärkter Platten bei Längsbelastungen zu untersuchen.

Wir weisen darauf hin dass es zwei grundlegende Methoden für die Untersuchung von gepressten Metallteilen gibt: diejenige der Gleichgewichtszweitelung, mit der die KRITISCHEN Belastungen der VOLLKOMMENEN Systeme bestimmt werden können, und diejenige der Gleichgewichtsabweichung, mit der die ERSCHOEPFUNGSBELASTUNGEN der REALEN Systeme festgestellt werden können. Der grundlegende Unterschied zwischen beiden Methoden beruht darauf, dass bei der ersteren die Beugung als ein indifferentes Gleichgewicht (unendliche Lösungen) untersucht wird, währenddem bei letzterer die Beugung als Festigkeitsproblem (Höchster Punkt des Last-Verformungsdiagramms) geprüft wird.

Wir untersuchen die Beugung der Platte indem wir dieselbe als ein Problem de Gleichgewichtsweiteilung betrachten. Infolgedessen nehmen wir an, dass die Aussenlasten vollkommen auf die Plattenmitte zentriert sind und vernachlässigen alle ihre geometrischen Unvollkommenheiten

In erster Linie untersuchen wir die elastisch Beugung dieser Struktursysteme. Darauf prüfen wir den Einfluss der Schweissrestspannungen auf den Wert der kleinsten kritischen Belastung unter der Annahme, dass es sich um eine elastiche oder elastoplastiche Beugung handelt. 
APENDICE 1 comportamiento placa (elástico)

$\beta_{n}$ $\rho_{n}$

\begin{tabular}{|c|c|c|}
\hline$N x>0$ & $\frac{n \pi}{1} \sqrt{\frac{1+\frac{n \pi}{1} \sqrt{\frac{N x}{D}+\left(\frac{n \pi}{1}\right)^{2}}}{2}}$ & $\frac{n \pi}{1} \sqrt{\frac{\frac{n \pi}{1} \sqrt{\frac{N x}{D}+\left(\frac{n \pi}{1}\right)^{2}-1}}{2}}$ \\
\hline$N x=0$ & $\frac{n \pi}{1}$ & $\frac{n \pi}{1} \sqrt{1-\frac{n \pi}{1} \sqrt{\frac{-N x}{D}}}$ \\
\hline$-N x<\left(\frac{n \pi}{1}\right)^{2} D$ & $\frac{n \pi}{1} \sqrt{1+\frac{n \pi}{1} \sqrt{\frac{N \pi}{D}}}$ & $\frac{n \pi}{1} \sqrt{2}$ \\
$-N x-\left(\frac{n \pi}{1}\right)^{2} D$ & $\frac{n \pi}{1} \sqrt{-1+\frac{n \pi}{1} \sqrt{-\frac{N x}{D}}}$ \\
\hline$-N x>\left(\frac{n \pi}{1}\right)^{2} D$ & $\frac{n \pi}{1} \sqrt{1+\frac{n \pi}{1} \sqrt{-\frac{N x}{D}}}$ & \\
\hline
\end{tabular}

comportamiento laja (elástico)

$\delta_{n}$ $Y_{n}$

\begin{tabular}{|c|c|c|}
\hline$N x=0$ & $\frac{n \pi}{1}$ & \\
\hline$N x=0$ & $\frac{n \pi}{1} \sqrt{1+\left(1-v^{2}\right) \frac{N x}{E t}}$ & $\frac{n \pi}{l} \sqrt{1+2(1+V) \frac{N x}{E t}}$ \\
\hline
\end{tabular}

APENDICE 2 comportamiento placa (plástico) $\beta_{n}$

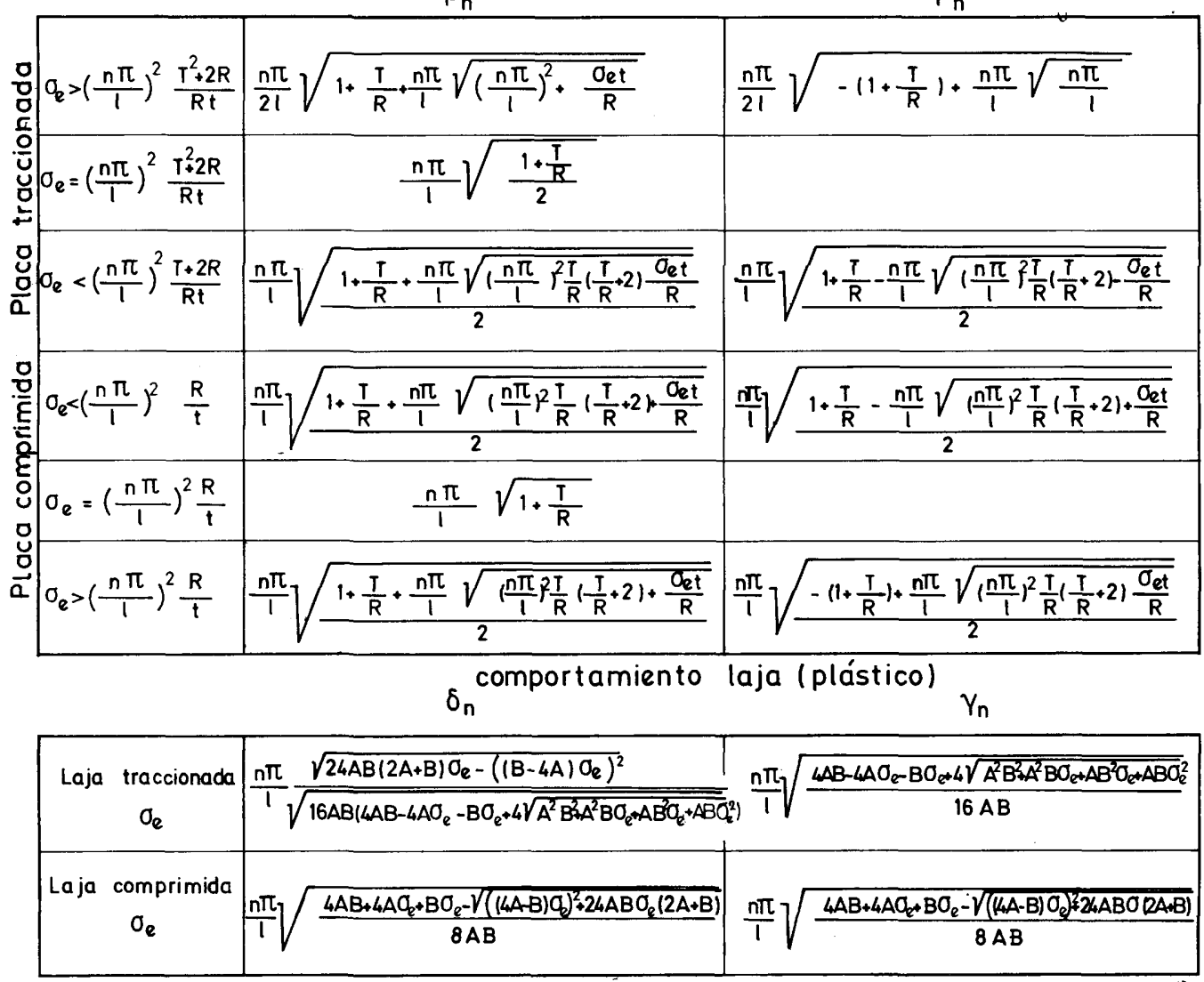




\section{APENDICE 3}

\section{Matriz de rigidez del comportamiento placa (elástico)}

\begin{tabular}{|c|c|c|c|c|c|c|c|}
\hline Caso & $p_{1,1}=p_{3,3}$ & $P_{1,2}=P_{2,1}=-P_{3,4}=-P_{4,3}$ & $p_{1,3}=p_{3,1}$ & $P_{1,4}=P_{L, 1}=-P_{2,3}=-P_{3,2}$ & $P_{2,2}=P_{4,4}$ & $P_{2,4}=P_{4,2}$ & \\
\hline$N x>0$ & $\beta \rho(\rho \operatorname{sh} 2 \beta b-\beta \operatorname{sen} 2 \rho b) / D_{1}$ & $\begin{array}{l}\left(\rho^{2} \sin ^{2} \beta b\left(\rho^{2}+\beta^{2}+\left(\frac{n \pi}{l}\right)^{2} v\right)+\right. \\
\left.+\beta^{2} \operatorname{sen}^{2} \rho D\left(\beta^{2}+\rho^{2}-\left(\frac{n \pi}{l}\right)^{2} v\right)\right) / D\end{array}$ & $2 \beta \rho(\beta \operatorname{ch} \beta b \operatorname{sen} \rho b-\rho s h \beta b \cos \rho b) / D_{1}$ & $\left(2 \beta \rho\left(\beta^{2}+\rho^{2}\right) \operatorname{sh} \beta b \operatorname{sen} \rho b\right) / D_{1}$ & $\beta \alpha\left(\beta^{2}+p^{2}\right)(\rho \operatorname{ssh} 2 \beta b+\beta \operatorname{sen} 2 \rho b) / D_{1}$ & $\begin{array}{c}-2 \beta p\left(\beta^{2}+\rho^{2}\right) \\
(\beta \operatorname{ch} \beta b \operatorname{sen} p b+\rho \operatorname{sh} \beta b \cos p b) / D_{1}\end{array}$ & $D_{1}=\frac{1}{D}\left((\rho \operatorname{sh} \beta b)^{2}-(\beta \operatorname{sen} \rho b)^{2}\right)$ \\
\hline$N x=0$ & $2 \beta(\operatorname{sh} \beta b \operatorname{ch} \beta b-\beta b) / D_{2}$ & $\left(-\beta^{2} \operatorname{sh}^{2} \beta b(1+V)+\beta^{4} D^{2}(V-1)\right) / D_{2}$ & $2 \beta(\beta b c h \beta b-\operatorname{sh} \beta b) / D_{2}$ & $\left(2 \beta^{3} b \operatorname{sh} \beta b\right) / D_{2}$ & $2 \beta^{3}(\beta b+\operatorname{sh} \beta b \operatorname{ch} \beta b) / D_{2}$ & $-2 \beta^{3}(\operatorname{sh} \beta b+\beta b \operatorname{ch} \beta b) / D_{2}$ & $D_{2}=\frac{1}{D}\left(\operatorname{sh}^{2} \beta b-\beta^{2} b^{2}\right)$ \\
\hline$-N x=<\left(\frac{n \pi}{1}\right)^{2} D$ & $\begin{array}{c}\left(\beta^{2}-\rho^{2}\right) \\
(\beta \operatorname{ch~} \beta b \operatorname{sh} \rho b-\rho \operatorname{ch} \rho b \operatorname{sh} \beta b) / D_{3}\end{array}$ & $\begin{array}{l}\left(2 \beta \rho\left(\frac{n \pi}{1}\right)^{2}(1-v)(1-\operatorname{cch} \beta b c h p b)+\right. \\
+2 \operatorname{sh} \beta b \operatorname{sh} \rho b\left(\beta^{2} \rho^{2}-\left(\frac{n \pi}{1}\right)^{4} v\right) / D_{3}\end{array}$ & $\left(\beta^{2}-p^{2}\right)(p \operatorname{sh} \beta b-\beta \operatorname{sh} \rho b) / D_{3}$ & $\beta \rho\left(\beta^{2}-\rho^{2}\right)(\operatorname{ch} \beta b-\operatorname{ch} \rho b) / D_{3}$ & $\begin{array}{c}\beta \rho\left(\beta^{2}-\rho^{2}\right) \\
(\beta \operatorname{sh} \beta b \operatorname{ch} \rho b-\rho \operatorname{ch} \beta b \operatorname{sh} \rho b) / D_{3}\end{array}$ & $\beta \rho\left(\beta^{2}-\rho^{2}\right)(\rho \operatorname{sh} \rho b-\beta s h \beta b) / D_{3}$ & $\begin{array}{l}D_{3}=\frac{1}{D}(2 \beta \rho(1-\operatorname{ch} \beta b c h p b)+ \\
\left.+\operatorname{sh} \beta b \operatorname{sen} \rho b\left(\beta^{2}+\rho^{2}\right)\right)\end{array}$ \\
\hline$-N x=\left(\frac{n \pi}{1}\right)^{2} D$ & $\beta(\operatorname{sh} \beta b-\beta b \operatorname{ch} \beta b) / D_{6}$ & $\begin{array}{l}\left(\beta^{2}(\operatorname{ch} \beta b-1)-v \frac{\beta^{2}}{2}\right. \\
(2 \operatorname{ch} \beta b-2-\beta b \operatorname{sh} \beta b 1) / D_{4}\end{array}$ & $\beta(\beta b-\operatorname{sh} \beta b) / D_{4}$ & $\beta^{2}(1-\operatorname{ch} \beta D) / D_{4}$ & $\left(-\beta^{3} \operatorname{sh} \beta b\right) / D_{4}$ & $\left(\beta^{3} \operatorname{sh} \beta b\right) / D_{4}$ & $D_{4}=\frac{1}{D} \beta(2 \operatorname{ch} \beta D-2-\beta D \operatorname{sh} \beta D)$ \\
\hline$-N x>\left(\frac{n \pi}{1}\right)^{2} D$ & $\begin{array}{c}\left(\beta^{2}+\rho^{2}\right) \\
(\beta \operatorname{cch} \beta b \operatorname{sen} \rho b-\rho \operatorname{sh} \beta b \cos \rho b) / D_{5}\end{array}$ & $\left(\begin{array}{l}2 \beta \rho\left(\frac{n \pi}{1}\right)^{2}(1-v)(1-\operatorname{ch} \beta b \cos \rho b)- \\
-2 \operatorname{sh} \beta b \operatorname{sen} \rho b\left(\beta^{2} \rho^{2}+\left(\frac{n \pi}{1}\right)^{4} v\right) / q_{5}\end{array}\right.$ & $\left(\beta^{2}+\rho^{2}\right)(\rho \operatorname{sh} \rho b-\beta$ sen $\rho b) / D_{5}$ & $\beta \rho\left(\beta^{2}+p^{2}\right)(\operatorname{ch} \beta b-\cos \rho b) / D_{5}$ & $\left|\begin{array}{c}\beta \rho\left(\beta^{2}+\rho^{2}\right) \\
(\rho \operatorname{cch} \beta b \operatorname{sen} \rho b+\beta \operatorname{ssh} \beta b \cos \rho b) / D_{5}\end{array}\right|$ & $-\beta \rho\left(\beta^{2}+\rho^{2}\right)(\beta \operatorname{sh} \beta b+\rho \operatorname{sen} \rho b) / D_{5}$ & $\begin{array}{l}D_{5}=\frac{1}{D}(2 \beta \rho(1-\operatorname{ch} \beta b \cos \rho b)+ \\
\left.+\operatorname{sh} \beta b \operatorname{sen} \rho b\left(\beta^{2}-\rho^{2}\right)\right)\end{array}$ \\
\hline
\end{tabular}

\section{Matriz de rigidez del comportamiento laja (elástico)}

\begin{tabular}{|c|c|c|c|c|c|c|c|}
\hline Caso & $I_{1,1}=I_{3,3}$ & $I_{1,2}=I_{2,1}=-I_{3,4}=-I_{4,3}$ & $l_{1,3}=I_{3,1}$ & $I_{1,4}=I_{4,1}=-I_{2,3}=-I_{3,2}$ & $I_{2,2}=I_{4,4}$ & $I_{2,4}=I_{4,2}$ & \\
\hline$N x=0$ & $((3-v) \operatorname{shn} 2 \delta b+2(1+v) \delta b) / D$ & $\left((1+v)^{2}(\delta b)^{2}-(3-v)(11-v) \operatorname{sh}^{2} \delta b\right) / D_{1}$ & $-2(13-v) \operatorname{sh} \delta \mathrm{b}+(1+\forall \delta b \operatorname{ch} \delta \mathrm{b}) / D_{1}$ & $(2(1+v) \delta b \operatorname{sh} \delta b) / D_{1}$ & $((3-v) \operatorname{sh} 2 \delta b-2(1+v) \delta b) / D_{1}$ & $2((1+v) \delta b \operatorname{ch} \delta b-(3-v) s \delta \delta b) / D_{1}$ & $\begin{array}{l}D_{1}=-\delta^{2} b^{2}\left(1+v^{2}\right)+ \\
\left.+(3-v)^{2} \operatorname{sh}^{2} \delta b\right) \frac{(1+v)}{E t \delta}\end{array}$ \\
\hline$N x \neq 0$ & $\begin{aligned} & \gamma\left(\operatorname{ch} \frac{\delta b}{2} \operatorname{ch} \frac{\gamma b}{2} / D_{2}+\right. \\
+ & \left.\operatorname{sh} \frac{\delta b}{2} \operatorname{sh} \frac{\gamma b}{2} / D_{3}\right)\end{aligned}$ & $\begin{array}{l}\frac{n \pi}{1}\left(\operatorname{ch} \frac{\delta b}{2} \operatorname{sh} \frac{\gamma b}{2} / D_{2^{+}}\right. \\
\left.+\operatorname{sh} \frac{\delta b}{2} \operatorname{ch} \frac{\gamma b}{2} / D_{3}-\frac{2}{(1+v)}\right)\end{array}$ & $\begin{array}{l}\gamma\left(\operatorname{sh} \frac{\delta b}{2} \operatorname{sh} \frac{\gamma b}{2} / D_{3}-\right. \\
\left.-\operatorname{ch} \frac{\delta b}{2} \operatorname{ch} \frac{\gamma b}{2} / D_{2}\right)\end{array}$ & $\begin{array}{l}\frac{n \pi}{1}\left(\operatorname{ch} \frac{\delta b}{2} \operatorname{sh} \frac{\gamma b}{2} / D_{2}-\right. \\
\left.-\operatorname{sh} \frac{\delta b}{2} \operatorname{ch} \frac{\gamma b}{2} / D_{3}\right)\end{array}$ & $\begin{array}{l}\delta\left(\operatorname{sh} \frac{\delta \mathrm{b}}{2} \operatorname{sh} \frac{\gamma \mathrm{b}}{2} / \mathrm{D}_{2}+\right. \\
\left.+\operatorname{ch} \frac{\delta \mathrm{b}}{2} \operatorname{ch} \frac{\gamma \mathrm{b}}{2} / \mathrm{D}_{3}\right)\end{array}$ & $\begin{array}{l}\delta\left(\operatorname{sh} \frac{\delta b}{2} \operatorname{sh} \frac{\gamma b}{2} / D_{2}-\right. \\
\left.-\operatorname{ch} \frac{\delta b}{2} \operatorname{ch} \frac{\gamma b}{2} / D_{3}\right)\end{array}$ & 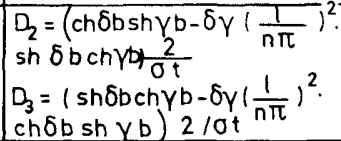 \\
\hline
\end{tabular}




\section{A PENDICE 4}

\section{Matriz de rigidez del comportamiento placa (plástico)}

\begin{tabular}{|c|c|c|c|c|c|c|c|c|}
\hline & Caso & $p_{1,1}^{\prime}=p_{3,3}^{\prime}$ & $P_{1,2}^{\prime}=P_{2,1}^{\prime} \quad P_{3,4}^{\prime}=-P_{4,3}^{\prime}$ & $P_{1,3}^{\prime}=P_{3,1}^{\prime}$ & $P_{1,4}^{\prime}=P_{4,1}^{\prime} \quad P_{2,3}^{\prime}=-P_{3,2}^{\prime}$ & $P_{2,2}^{\prime}=P_{4,4}^{\prime}$ & $P_{2,4}^{\prime}=P_{4,2}^{\prime}$ & \\
\hline $0^{\circ}$ & $a_{e}>\left(\frac{n \pi}{1}\right)^{2} \frac{T^{2}+2 R}{R t}$ & $\beta \rho(\rho \operatorname{sh} 2 \beta b-\beta \operatorname{sen} 2 \rho b) / D_{1}$ & $\begin{array}{l}-\left[\rho^{2} \operatorname{sh} \rho p\left(\rho^{2}+\beta^{2}+\left(\frac{n \pi}{l}\right)^{2} / 2\right)+\right. \\
\left.+\beta^{2} \operatorname{sen}^{2} \rho b\left(\beta^{2}+\rho^{2}-\left(\frac{n \pi}{1}\right)^{2} / 2\right)\right] / D_{1}\end{array}$ & $2 \beta \rho(\beta \operatorname{ch} \beta b \operatorname{sen} p b \cdot \rho \operatorname{sh} \beta b \cos \rho b) / D_{1}$ & $\left(2 \beta D\left(\beta^{2}+\rho^{2}\right) \operatorname{sh} \beta b \operatorname{sen} \rho b\right) / D_{1}$ & $\beta \rho\left(\beta^{2}+p^{2}\right)(\rho \operatorname{ss} 2 \beta b+\beta \operatorname{sen} 2 \rho b) / D_{1}$ & $\begin{array}{c}-2 \beta \rho\left(\beta^{2}+\rho^{2}\right) \\
(\beta \operatorname{ch} \beta b \operatorname{sen} p b+\rho \operatorname{sh} \beta b \cos \rho b) / D_{1}\end{array}$ & $D_{1}=\frac{1}{4 R}\left((\rho \operatorname{sh} \beta b)^{2}-(\beta \operatorname{sen} \rho b)^{2}\right)$ \\
\hline 范 & $\sigma_{e}=\left(\frac{n \pi}{1}\right) \frac{T^{2}+2 R}{R t}$ & $2 \beta(\operatorname{sh} \beta b \operatorname{ch} \beta b-\beta b) / D_{2}$ & $-\left(\frac{n \pi)^{2}}{1}\left[1+\frac{T}{2 R}\right) \operatorname{sh}^{2} \beta b+\frac{T}{2 R} \beta^{2} b^{2}\right] / D_{2}$ & $2 \beta(\beta b \operatorname{ch} \beta b-\operatorname{sh} \beta b) / D_{2}$ & $\left(2 \beta^{3} b \operatorname{sh} \beta b\right) / D_{2}$ & $2 \beta^{3}(\beta b+\operatorname{sh} \beta b \operatorname{ch} \beta b) / D_{2}$ & $-2 \beta^{3}(\operatorname{sh} \beta b+\beta b \operatorname{ch} \beta b) / D_{2}$ & $D_{2}=\frac{1}{4 R}\left(s h^{2} \beta b-\beta^{2} b^{2}\right)$ \\
\hline$\stackrel{g}{0}$ & $a_{e}<\left(\frac{n \pi}{1}\right) \frac{t^{2}+2 R}{R t}$ & $\begin{array}{c}\left(\beta^{2}-\rho^{2}\right) . \\
(\beta \operatorname{ch} \beta \operatorname{ssh} \rho b-\rho \operatorname{ch} \rho b \operatorname{sh} \beta b) / D_{3}\end{array}$ & $\begin{array}{l}{\left[\frac{T}{R} \beta \rho\left(\frac{n \pi}{l}\right)^{2}(1-\operatorname{ch} \beta b c h \rho b)+\right.} \\
\left.+2 \operatorname{sh} \beta b \operatorname{shpb}\left(\beta^{2} \rho^{2}-\left(\frac{n \pi}{l}\right) \frac{\left(1+\frac{T}{R}\right)}{L}\right)\right] M_{3}\end{array}$ & $\left.\left(\beta^{2}-\rho^{2}\right) \rho \operatorname{sh} \beta b-\beta \operatorname{sh} \rho b\right) / D_{3}$ & $\beta \rho\left(\beta^{2}-\rho^{2}\right)(\operatorname{ch} \beta b-\operatorname{ch} \rho b) / D_{3}$ & $\begin{array}{c}\beta \rho\left(\beta^{2}-\rho^{2}\right) \\
\left(\beta \operatorname{sh} \beta b \operatorname{ch} \rho b-\rho \operatorname{ch} \beta b \operatorname{ssh} \rho b / D_{3}\right.\end{array}$ & $\beta \rho\left(\beta^{2}-\rho^{2}\right)(\rho s h \rho b-\beta s h \beta b) / D_{3}$ & $\begin{array}{l}D_{3}=\frac{1}{4 R}(2 \beta \rho(1-\operatorname{ch} \beta b c h p b)+ \\
\left.+\operatorname{sh} \beta b \operatorname{sen} \rho b\left(\beta^{2}+\rho^{2}\right)\right)\end{array}$ \\
\hline $0^{\circ}$ & $a_{e}<\left(\frac{n \pi}{1}\right)^{2} \frac{R}{t}$ & $\begin{array}{c}\left(\beta^{2}-\rho^{2}\right) . \\
\cdot\left(\beta \operatorname{ch} \beta b \operatorname{sh} \rho b-\rho \operatorname{ch} \rho b \operatorname{sh} \beta b / / D_{3}\right.\end{array}$ & $\begin{array}{l}{\left[\frac{T}{R} \beta \rho\left(\frac{n \pi}{1}\right)^{2}(1-\operatorname{ch} \beta b c h \rho b)+\right.} \\
+2 \operatorname{sh} \beta b \operatorname{shpb}\left(\beta^{2} \rho^{2}-\left(\frac{n \pi \pi^{4}}{T}\right)\left(1+\frac{T}{4}\right)\right. \\
\end{array}$ & $\left(\beta^{2}-\rho^{2}\right)(\rho \operatorname{sh} \beta b-\beta \operatorname{sh} \rho b) / D_{3}$ & $\beta \rho\left(\beta^{2}-\rho^{2}\right)(\operatorname{ch} \beta b-\operatorname{ch} \rho b) / D_{3}$ & $\begin{array}{c}\beta \rho\left(\beta^{2}-\rho^{2}\right) \\
(\beta \operatorname{sh} \beta b \operatorname{ch} \rho b-\rho \operatorname{ch} \beta b \operatorname{sh} \rho b) / D_{3}\end{array}$ & $\beta \rho\left(\beta^{2}-\rho^{2}\right)(\rho s h \rho b-\beta s h \beta b) / D_{3}$ & $\begin{array}{l}D_{3}=\frac{1}{4 R}(2 \beta \rho(1-\operatorname{ch} \beta b \operatorname{ch} \rho b)+ \\
\left.+\operatorname{sh} \beta b \operatorname{sen} \rho b\left(\beta^{2}+\rho^{2}\right)\right)\end{array}$ \\
\hline 'a & $\sigma_{e}=\left(\frac{n \pi}{1}\right)^{2} \frac{R}{t}$ & $\beta(\operatorname{sh} \beta b-\beta b \operatorname{ch} \beta b) / D_{4}$ & $\left(\frac{n \pi}{1}\right)\left[\frac{T}{R}(\operatorname{ch} \beta \mathrm{b}-1)+\frac{\beta \mathrm{b}}{2} \operatorname{sh} \beta \mathrm{b}\right] / \mathrm{D}_{4}$ & $\beta(\beta b-\operatorname{sh} \beta b) / D_{4}$ & $\beta^{2}(1-\operatorname{ch} \beta b) / D_{6}$ & $\left(-\beta^{3} \operatorname{sh} \beta b\right) / D_{4}$ & $\left(\beta^{3} \operatorname{sh} \beta b\right) / D_{4}$ & $D_{L}=\frac{1}{4 R} \beta(2 \operatorname{cch} \beta b-2-\beta b \operatorname{sh} \beta b)$ \\
\hline g & $\sigma_{e}>\left(\frac{n \pi}{l}\right)^{2} \frac{R}{t}$ & $\begin{array}{c}\left(\beta^{2}+\rho^{2}\right) \\
(\beta \operatorname{ch} \beta b \operatorname{sen} \rho b-\rho \operatorname{sh} \beta b \cos \rho b) / D_{5}\end{array}$ & $\begin{array}{l}{\left[\frac{I}{R} \beta \rho\left(\frac{n \pi}{T}\right)^{2}(1-\operatorname{ch} \beta b \cos p b)-\right.} \\
\left.\left.-2 \sin \beta b \operatorname{sen} p b \beta^{2} p^{2}+\left(\frac{n \pi}{1}\right)^{4} \frac{\left.1+\frac{T}{R}\right)}{4}\right)\right] / D_{5}\end{array}$ & $\left(\beta^{2}+p^{2}\right)\left(p s h p b-\beta\right.$ senpb) $/ D_{5}$ & $\beta p\left(\beta^{2}+p^{2}\right)(\operatorname{cch} \beta b-\cos \rho b) / D_{5}$ & $\begin{array}{c}\beta \rho\left(\beta^{2}+\rho^{2}\right) \\
(\rho \operatorname{cch} \beta b \operatorname{sen} \rho b+\beta \operatorname{shh} \beta b \cos \rho b) / D_{5}\end{array}$ & $-\beta \rho\left(\beta^{2}+\rho^{2}\right)(\beta \operatorname{sh} \beta b+\rho \operatorname{sen} \rho b) / D_{5}$ & $\begin{array}{l}D_{5}=\frac{1}{4 R}(2 \beta \rho(1-\operatorname{ch} \beta b \cos \rho b)+ \\
\left.+\operatorname{sh} \beta b \operatorname{sen} \rho b\left(\beta^{2}-\rho^{2}\right)\right)\end{array}$ \\
\hline
\end{tabular}

\section{Matriz de rigidez del comportamiento laja (plástico)}

\begin{tabular}{|c|c|c|c|c|c|c|c|}
\hline Caso & $l_{1,1}^{\prime}=l_{3,3}^{\prime}$ & $i_{1,2}=i_{3,1}^{\prime}=-i_{3,4}^{\prime}=-i_{4,3}^{\prime}$ & $i_{1,3}^{\prime}=I_{3,1}^{\prime}$ & $I_{1,4}^{\prime}=I_{4,1}^{\prime}=-I_{2,3}^{\prime}=-I_{3,2}^{\prime}$ & $i_{2,2}^{\prime}=i_{4,4}^{\prime}$ & $i_{2,4}^{\prime}=i_{4,2}^{\prime}$ & \\
\hline $\begin{array}{c}\text { Laja traccionada } \\
\sigma_{e}\end{array}$ & $\begin{array}{l}\frac{\delta \gamma}{2}(g-f) \\
(\delta f \operatorname{sen} 2 \gamma b+\gamma g \operatorname{sh} 2 \delta b) / D_{1}\end{array}$ & $\begin{array}{l}-\frac{B}{4 A}\left(\gamma^{2} g^{2} \operatorname{sh}^{2} \delta b\left(\frac{n \pi}{1}-1\right)-\right. \\
\left.-\delta^{2} f^{2} \operatorname{sen}^{2} \gamma b\left(1+\frac{n \pi}{1}\right)\right) / D_{1}\end{array}$ & $\begin{array}{c}\delta \gamma(f-g) . \\
.\left(\delta f \operatorname{ch} \delta b \operatorname{sen} \gamma b+\gamma g \operatorname{sh} \delta b \cos \gamma b / D_{1}\right.\end{array}$ & $\begin{array}{c}\delta \gamma(g-f) . \\
\left.\left(\delta^{2} f^{2}+y^{2} g^{2}\right) \operatorname{sh} \delta b \operatorname{sen} \gamma b\right) / D_{1}\end{array}$ & $\begin{array}{c}\frac{B \delta \gamma}{8 A}(f+g) \\
\cdot(y g \operatorname{sh} 2 \delta b-\delta f \operatorname{sen} 2 \gamma b) / D_{1}\end{array}$ & $\begin{array}{c}\frac{B}{4 A} \delta \gamma(f+g) \\
\left(\delta f \operatorname{cc} \delta b \operatorname{sen} \gamma b-\gamma g c \delta b \cos \gamma b / D_{1}\right.\end{array}$ & $D_{1}=$ \\
\hline $\begin{array}{l}\text { Laja comprimida } \\
\qquad a_{e}\end{array}$ & $\begin{array}{c}(f \delta-g \gamma) . \\
\cdot(g \operatorname{sen} \delta b \cos \gamma b \cdot \operatorname{sen} \gamma b \cos \delta b) / D_{2}\end{array}$ & $\begin{array}{l}-\left(f g\left(f \delta+g \gamma-\frac{n \pi}{1}\right)(1-\cos \delta b \cos \gamma b)+\right. \\
+\left(\frac{f^{2}+g^{2}}{2} \frac{n \pi}{1}-f g(\delta g+f \gamma) \operatorname{sen} \delta b \operatorname{sen} \gamma b\right) / 2\end{array}$ & $(f \delta-g \gamma)(f \operatorname{sen} \gamma b-g \operatorname{sen} \delta b) / D_{2}$ & fg $(f \delta-g \gamma)(\cos \gamma b-\cos \delta b) / D_{2}$ & $\begin{array}{c}\frac{B}{4 A}(f \gamma-g \delta) \cdot \\
\cdot(g \operatorname{sen} \gamma b \cos \delta b-f \operatorname{sen} \delta b \cos \gamma b) / D_{2}\end{array}$ & $\begin{array}{c}\frac{B}{4 A}(f \gamma-g \delta) \\
(f \operatorname{sen} \delta b-g \operatorname{sen} \gamma b) / D_{2}\end{array}$ & $\begin{array}{l}D_{2}=(2 f g(1-\cos \delta b \cos \gamma b)- \\
\left.-\operatorname{sen} \delta b \operatorname{sen} \gamma b\left(f^{2}+g^{2}\right)\right) / 4 A t\end{array}$ \\
\hline
\end{tabular}


(1) WILLTAMS, F.W. and WITTRICK, W.H. "Computational procedures for a matrix analysis of the stability and vibration of thin flat-wa eled structures in compressión" International Journal of Mechani cal Sciences, vol.2 (1969).

(2) WILLIAMS, F.W. and WITTRICK, W.H. "Numerical results for the ini tial buckling of some sitiffened panels in compression" Aeronautical quarterly, vol, 23 (1972).

(3) SMITH, C.S. "Beding, buckling and vibration of orthotropic plate-beam structures" Journal of Ship Research, Vol. 12 11968).

(4) SMITH, C.S. "Buckling problems in the design of fibreglass-reiforcement plastic ships" Journal of Ship Research, vol. 16 (1972).

(5) PREZEMIENIECKI, J.S. "Matrix analys is of local instability in plates, stiffened panels and columns" International Journal for Numerical Methods in Engineering vol. 5 (1972).

(6) PLANK, R.J. and WITTRICK, W.H. "Buckling under combined loading of thin, flat-walled structures by a complex finite strip method" International Journal for Numerical Methods in Engineering (1974).

(7) FUKUMOTO, Y., USAMI, T. and OKAMOTO, Y. "Ultimate strength of stif fened plates" The ASCE Specialty Conference on Metal Bridge, St. Louis $(1974)$.

.8) FUKUMOTO, Y., USAMI, T. and YAMAGUCHI, K. "Inelastic buckling strength of stiffened plates in compression" IABSE P-8 (1977).

(9) HASEGAWA, A., OTA, K. and NISHINO, F. "Buckling strength of mul tiple stiffened plates" Methods of Structural Analysis, University of Wisconsin-Madison (1976).

(10)CHAJES, A. "Principles of structural stability theory" Prentice Hall (1974).

(11) NOVOZHILOV, V.V. "Foundations of the nonlinear theory of elasti city" Graylock Press, Rochester, N.V. (1953).

(12)WITTRICK, W.H. and WILLIAMS, F.W. "An algorithm for computing critical buckling loads of elastic structures" Journal of Struc tural Mechanics, vol. 1 (1973).

(13)BIJLARD, P.P. "Theory and test on the plastic stability of plates an shells" Journal of the Aeronautical Sciences, vol. 16 (1949). 